by

\title{
Tianyi Qiu
}

B.S, Hunan University, 2014

\author{
Submitted to the Graduate Faculty of \\ Swanson School of Engineering in partial fulfillment \\ of the requirements for the degree of \\ Master of Science
}

University of Pittsburgh 


\section{UNIVERSITY OF PITTSBURGH SWANSON SCHOOL OF ENGINEERING}

This thesis was presented

by

Tianyi Qiu

It was defended on

March 31, 2016

and approved by

Nitin Sharma, PhD, Assistant Professor

Department of Mechanical Engineering and Materials Science

William W. Clark, PhD, Professor

Department of Mechanical Engineering and Materials Science

Zhi-Hong Mao, PhD, Associate Professor

Department of Electrical and Computer Engineering

Thesis Advisor: Nitin Sharma, PhD, Assistant Professor 
Copyright (C) by Tianyi Qiu

2016 


\title{
ROBUST COMPENSATION OF ELECTROMECHANICAL DELAY DURING NEUROMUSCULAR ELECTRICAL STIMULATION OF ANTAGONISTIC MUSCLES
}

\author{
Tianyi Qiu, M.S.
}

University of Pittsburgh, 2016

Neuromuscular electrical stimulation (NMES) can potentially be used to restore the limb function in persons with neurological disorders, such as spinal cord injury (SCI), stroke, etc.

Researches on control system design has so far focused on relatively simple unidirectional NMES applications requiring stimulation of single muscle group. However, for some advanced tasks such as pedaling or walking, stimulation of multiple muscles is required. For example, to extend as well as flex a limb joint requires electrical stimulation of an antagonistic muscle pair. This is due to the fact that muscles are unidirectional actuators. The control challenge is to allocate control inputs to antagonist muscles based on the system output, usually a limb angle error to achieve a smooth and precise transition between antagonistic muscles without causing discontinuities. Furthermore, NMES input to each muscle is delayed by an electromechanical delay (EMD), which arises due to the time lag between the electrical 
excitation and the force development in muscle. And EMD is known to cause instability or performance loss during closed-loop control of NMES.

In this thesis, a robust delay compensation controller for EMDs in antagonistic muscles is presented. A Lyapunov stability analysis yields uniformly ultimately bounded tracking for a human limb joint actuated by antagonistic muscles. The simulation results indicate that the controller is robust and effective in switching between antagonistic muscles and compensating EMDs during a simulated NMES task. Further experiments on a dual motors testbed shows its feasibility as an NMES controller for human antagonistic muscles. 
TABLE OF CONTENTS

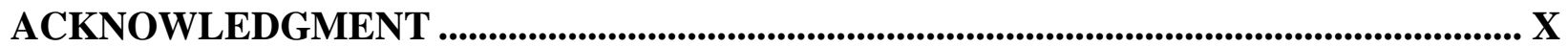

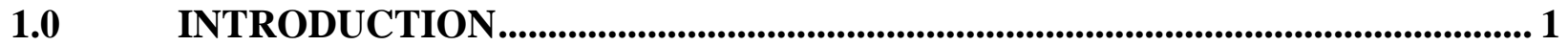

2.0 BACKGROUND INFORMATION AND LITERATURE REVIEW .....................5

CONTROLLER DEVELOPMENT ...................................................................... 11

MUSCULOSKELETAL SYSTEM....................................................................... 11

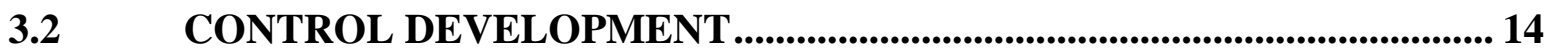

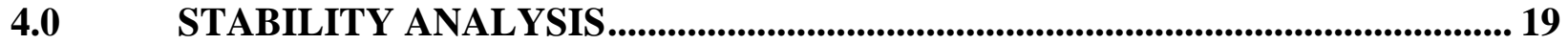

5.0 SIMULATIONS \& EXPERIMENTS.................................................................. 24

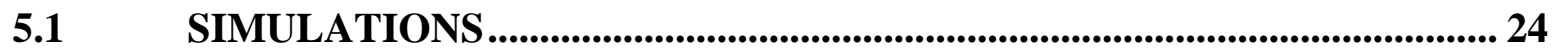

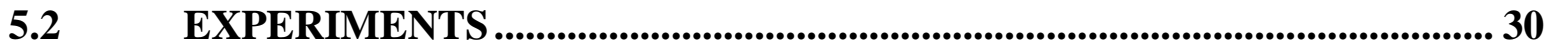

5.2.1 Single Motor Experiment ................................................................. 30

5.2.2 Dual Motors Experiment............................................................................. 35

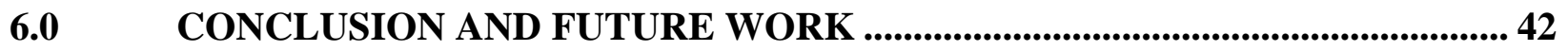

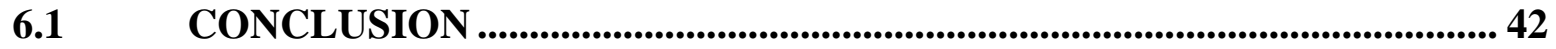

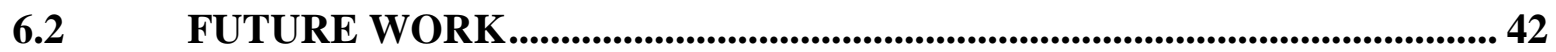

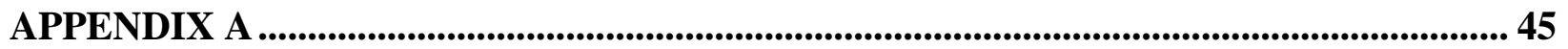

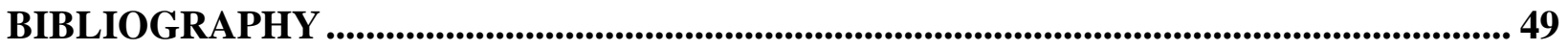




\section{LIST OF TABLES}

Table 1. RMSE of Simulation with Different EMD Value ................................................... 29

Table 2. RMSE of Single Motor Experiments with Different EMD Value............................... 35

Table 3. RMSE of Experiments and Simulations with Different EMD Value ......................... 40 


\section{LIST OF FIGURES}

Figure 1. ERGYS 3 Rehabilitation System.................................................................. 7

Figure 2. Trajectory and Normalized Stimulation (u1,u2) Plots from Simulation ..................... 25

Figure 3. Normalized Error and Control Input ( v ) Plots from Simulation ............................... 25

Figure 4. Trajectory and Normalized Stimulation (u1,u2) Plot from Simulation (75ms) ........... 27

Figure 5. Normalized Error and Control Input ( v ) Plot from Simulation (75ms)..................... 27

Figure 6. Trajectory and Normalized Stimulation Plot (u1,u2) from Simulation (85ms) ........... 28

Figure 7. Normalized Error and Control Input ( v ) Plot from Simulation (85ms).................... 29

Figure 8. Trajectory and Motor Input (u1,u2) Plot from Single Motor Experiment ................... 31

Figure 9. Error and Control Input Plot ( v ) from Single Motor Experiment ............................. 32

Figure 10. Trajectory and Motor Input (u1,u2) Plot from Single Motor Experiment (75ms) ...... 33

Figure 11. Error and Control Input Plot ( v ) from Single Motor Experiment (75ms) ................ 33

Figure 12. Trajectory and Motor Input (u1,u2) Plot from Single Motor Experiment (85ms) ...... 34

Figure 13. Error and Control Input Plot ( v ) from Single Motor Experiment (85ms) ................ 34

Figure 14. Dual Motors Experiment Testbed .................................................................. 36

Figure 15. Trajectory and Motor Input (u1,u2) Plot from Dual Motors Experiment .................. 37

Figure 16. Error and Control Input ( v ) Plot from Dual Motors Experiment ........................... 37 
Figure 17. Trajectory and Motor Input (u1,u2) Plot from Dual Motors Experiment (75ms) ....... 38

Figure 18. Error and Control Input ( v ) Plot from Dual Motors Experiment (75ms) ................ 39

Figure 19. Trajectory and Motor Input $(\mathrm{u} 1, \mathrm{u} 2)$ Plot from Dual Motors Experiment (85ms) ....... 39

Figure 20. Error and Control Input ( v ) Plot from Dual Motors Experiment (85ms) ................ 40

Figure 21. Demonstration of Planned Human NMES Experiments 1 ...................................... 43

Figure 22. Demonstration of Planned Human NMES Experiments 2 ................................... 43

Figure 23. Simulink Block Diagram of Controller ......................................................... 45

Figure 24. Simulink Block Diagram for Simulation.......................................................... 46

Figure 25. Simulink Block Diagram for Single Motor Experiment ....................................... 47

Figure 26. Simulink Block Diagram for Dual Motors Experiment ........................................ 48 


\section{ACKNOWLEDGMENT}

I would like to give great and sincere thanks to my advisor, Prof. Nitin Sharma for his patience, generousness and considerable help throughout my study and research. Without his guidance and support, I would have never accomplished my research.

I would also thank my committee members: Prof. William Clark, Prof. Zhi-Hong Mao and Prof. Nitin Sharma for their time to make this thesis better.

I would have my thanks to lab members in Dr. Sharma’s research group: Naji Alibeji and Dr. Nicholas Kirsch for sharing priceless experience on research as well as experiments. My thanks also go to my lab partners: Qiang Zhong, Xuefeng Bao for their kind help.

Finally, I would thank my family for supports and encouragements. 


\subsection{INTRODUCTION}

Neuromuscular electrical stimulation (NMES) is commonly prescribed to treat or recover lost muscle function and/or strength in individuals who have upper motor neuron disorders from spinal cord injury (SCI), traumatic brain injury, multiple sclerosis, stroke, etc [1]. NMES artificially contracts muscle groups by applying external stimulation current. Closed-loop control of NMES can be used to generate advanced functional tasks (in this case NMES is also called functional electrical stimulation (FES)) such as walking [2, 3], single leg extension [4-9], and upper extremity grasping and reaching [10-12].

Linear control methods are not capable of more advanced and complicated NMES tasks and do not guarantee stability due to the presence of uncertainty and nonlinearity in the musculoskeletal system, and other causes such as external loads, muscle fatigue, electromechanical delay (EMD). Examples of linear control methods include proportional integral derivative (PD/PID), linear quadratic Gaussian control, pole placement method, gain scheduling control method [13-15]. Nonlinear control methods [4-9, 16-18] have also been recently developed for NMES. These nonlinear methods have improved performance over linear control methods. Robust nonlinear control of NMES is especially more relevant, and controllers have been developed to compensate for the nonlinear and uncertain muscles dynamics [5, 7], time-varying phenomena such as muscle fatigue [19], and electromechanical delay [18, 19]. 
Electromechanical delay (EMD) is the time lag between the electrical excitation and the force development in muscle. In results such as $[4,6,19]$, the EMD was modeled as an input delay in the musculoskeletal dynamics. Input delays can cause performance degradation and system instability, such as during human stance experiments [20]. Recently, a robust compensation control method was developed for an uncertain input delayed system with additive disturbances [21] and it suggests that a PD/PID controller can be augmented with a delay compensator that contains a finite integral of past control values to transform the delayed system into a delay-free system. Modified PD/PID controllers have been extended to compensate for EMD during NMES $[18,19]$.

However, these controllers are designed for unidirectional limb movements; i.e., only quadriceps muscles were stimulated through a single NMES channel to extend the lower leg. The antagonist muscle (hamstrings in this case) were not stimulated instead the leg is lowered by a controlled reduction in NMES of the quadriceps muscle allowing gravity to help bring the leg back towards the equilibrium position. However, in a standing position or during a gait cycle, producing knee flexion and extension would require controlled stimulation of both hamstring and quadriceps (antagonistic pair). Similarly, upper-limb movements such as elbow or wrist flexion and extension would require FES of antagonistic pairs (e.g., biceps and triceps muscles). In recent years, iterative learning control (ILC) was used for FES applications for upper limbs [2225]. In these studies, the stimulation was applied to triceps and anterior-deltoids muscles. However, the closed-loop control of the stimulation allowed only unidirectional joint movement like the flexion or extension of the muscles. This implies the controller may not be capable of controlling antagonistic muscles to flex as well as extend an upper-limb joint in a smooth and relative more efficient way. 
The field of multi-channel FES control is relatively new. In Downey et al. [9], asynchronous NMES control of the multiple muscles in the quadriceps muscle group was proposed through four channels. The switching between the channels is based on a fixed time periodic signal in order to reduce the onset of muscle fatigue. Again, this switching scheme was only proposed for agonist muscles. In addition, the switching signal was periodic and fixed. In order to design a controller for multi-channel control of antagonist muscle pairs, the switching signal would have to be based on the performance of the controller (i.e., the tracking error).

The primary focus of this thesis is to develop a closed-loop tracking controller for dual control of NMES tasks such as limb extension and flexion. A proportional-derivative (PD)-type closed-loop controller with delay compensation (DC) to deal with the EMD was designed for stimulating antagonistic muscle pairs in a musculoskeletal system. Unlike previous controllers that produce joint flexion with the help of gravity $[9,18,19]$ or controlled by correctional forces such as robot arms [22, 24], the developed controller will flex and extend a limb joint by stimulating agonist and antagonist muscles. The controller is designed to transition smoothly between the stimulation of the antagonist muscles. The switching signal is based on the position tracking error and can be made arbitrarily fast or slow by adjusting the control gains. Parametric uncertainty and additive bounded disturbance were included in the dynamics for the control development and subsequent stability analysis. Lyapunov Krasovskii (LK) functionals were constructed to cancel the delay terms. The associated Lyapunov-based stability analysis proved semi-global uniformly ultimately bounded tracking.

The thesis is organized as follows: Chapter 1 is a brief introduction to the development of FES research and the novelties of the research in this thesis. The second chapter features the background information of FES, EMD, and literature review on NMES control design and 
control methods for antagonistic muscle groups. The musculoskeletal system used for developing the controller is discussed in Chapter 3 and in Chapter 4 the design of the antagonistic controller with delay compensation is presented and its stability is proved through Lyapunov-based stability analysis. The simulation and experiment results are shown in Chapter 5 . The last chapter concludes the thesis and gives a few ideas for future work.

The main contributions of this thesis is listed below:

Chapter 3: Main contribution is to introduce the switching signals for transition antagonistic muscle and auxiliary terms to compensate for EMD.

Chapter 4: Main contribution is to yield that the controller is semi-globally uniformly ultimately bounded.

Chapter 5: Main contribution is to verify the controller through simulations and experiments on motor testbed. 


\subsection{BACKGROUND INFORMATION AND LITERATURE REVIEW}

Neuromuscular electrical stimulation (NMES) has been used to recover muscle function in individuals, who have partial or complete loss of limb control, due to trauma to upper nervous system systems or neurological disorders [1]. It can be used as an assistive therapy for relearning motor tasks method or as primary treatment to restore limb function. Some studies and clinical reviews have approved its effectiveness to recover motor function [26-28].

NMES achieves the control of muscle movement by using external low-level electrical current. During NMES, the motorization cell's membrane potential is depolarized, which releases calcium ions to be released from the sarcoplasmic reticulum. Causes calcium spark to activates the myofilaments, thus allow cross-bridge cycling that produces muscle force [29]. The external current is sent via the electrodes to the muscle. Electrodes can be transcutaneous (placed on the skin surface), percutaneous (placed within a muscle), epimysial (placed on the surface of the muscle), or cuff (wrapped around the nerve that innervates the muscle of interest) [30]. Skin surface electrodes are widely adopted in the commercial and clinical environment due to their wearability (easy to apply on and take off from the skin) and less invasive characteristic compared to other kinds of electrodes [31].

One of the most common application for NMES is to restore the motor functions for people who suffer from spinal cord injury (SCI), which is caused by either injury or trauma to the neurological tissue of the spinal cord. Approximately 400,000 people in America are 
currently living with SCI and the number is increasing at the rate of approximately 11,000 per year [32]. This irreversible damage can result in partial or total loss of sensory function, or paralysis, to parts of the body below the level of the injury, which affect patients' life quality dramatically [33]. NMES can be used to improve their motor functions, and thus restore their ability to perform daily tasks.

Another major field of NMES application is for the rehabilitation of stroke patients. During stroke the brain cells are damaged or die due to lack of blood supply. The stroke leads to the loss of motor function, especially of upper extremity. During 3 to 6 months after stroke, only $50 \%$ of stroke survivors are likely to regain some functional use of their affected upper extremity [34].There are several treatment methods for stroke patients to regain the motor function, such as Bobath therapy [35], constraint-induced therapy [36], task-specific motor relearning program [37] and robotic training [38]. Compared to traditional therapy, NMES methods have proven their effectiveness such as increasing movement and activity compared to traditional training alone [39]. The effectiveness of NMES in improving the muscle strength and the motor function recovery has been widely accepted by both clinical reviews and meta-analyses [27, 40, 41]. Besides, when combined with traditional motor training, the clinical NMES treatment will cost significantly less than using robotic therapy, the difference can be up to $\$ 1,000$ per patient while achieving the same degree of recovery [42].

A variety of NMES devices is commercially available due to its proven effectiveness. Most NMES systems in clinics use open-loop or finite state control systems. Foot drop correction devices such as L300 from BIONESS, Inc. help patient by monitoring whether the user's heel lifts off the ground through a sensor and stimulate the flexor muscles of the ankle. In this way, the user will be able to swing the leg and take a step. 
Besides open-loop and finite-state NMES systems, closed loop NMES systems are also available for more advanced tasks. NMES cycling machine such as the RT300 from Restorative Therapies, Inc. and Ergys 2 from Therapeutic Alliances, Inc. implement a closed-loop controller to maintain a constant cycling cadence by increasing the stimulation intensity as the muscles begin to fatigue.

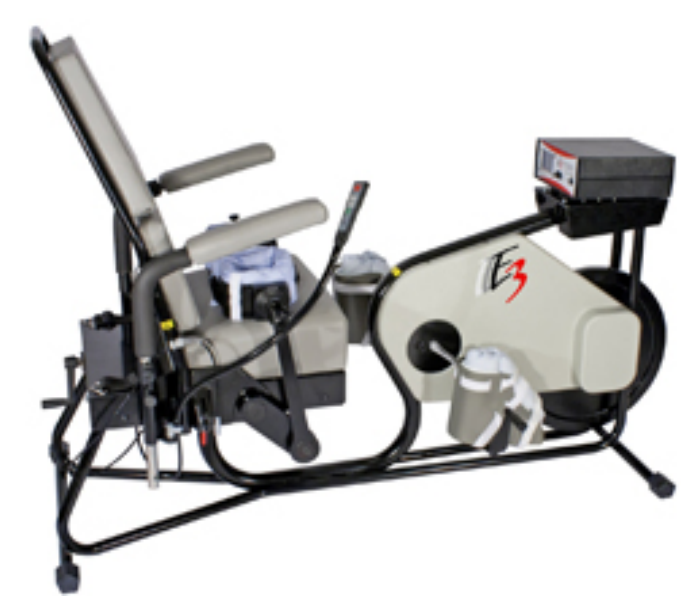

Figure 1. ERGYS 3 Rehabilitation System

Linear control methods for NMES applications include proportional integral derivative (PD/PID), linear quadratic Gaussian control, pole placement method, gain scheduling control [13-15]. However, because the musculoskeletal system is uncertain and nonlinear, and various external disturbances such as loads on muscle, muscle fatigue, and electromechanical delay (EMD) could lead the system to instability. These control methods are neither capable of advanced and more complicated NMES tasks that require real-time control of the stimulation, nor they can guarantee stability.

In order to achieve better performance than linear closed-loop controller, some nonlinear control methods like sliding mode control, neural network, nonlinear model predictive control 
have been introduced for NMES application. These nonlinear methods were proved to have enhanced performance over linear control methods in previous researches [4-9, 16-18]. Among these nonlinear control techniques, robust nonlinear control of NMES in [5, 7] is especially more relevant given the nonlinear and uncertain muscle dynamics, time-varying phenomena such as muscle fatigue [19], and electromechanical delay [18, 19].

EMD is the time window between electrical excitation of the motor-neurons and development of contraction in muscle. It is a function of a number of phenomena including finite propagation time of the chemical ions in the muscle, cross-bridge formation between actinmyosin filaments, stretching of the series elastic components in response to the external electrical input, synaptic transmission delays, and others [43], [44]. It is modeled as an input delay in the musculoskeletal dynamics in results such as $[4,6,19]$. EMD is a crucial factor in muscle model used for NMES controller design since these delays can easily degrade the performance and affect the stability of the system, make the whole system unstable. [44]

Even though the EMD is one of the major problems in NMES control technology, not too many researchers had taken EMD into consideration during the controller design process. Few mathematical tools exist for compensating input delay; they are Smith predictors [45], finite spectrum assignment [46] and Artstein model reduction [47]. Recently, a robust compensation control method was developed for an uncertain input delayed system with additive disturbances in [21]. The study shows a model-free robust controller that could make the leg shank to track the desired trajectory under known constant EMD with a uniformly ultimately bounded error. Also, the study suggests a PD/PID controller with a delay compensator that contains a finite integral of past control values can transform the delayed system into a delay-free system. Modified PD/PID controllers have been extended to compensate for time varying EMD during 
NMES $[18,19]$. In order to achieve precise and efficient controlled contraction in NMES, EMD should be taken into account during the control development. Similar to results in $[4,6]$, in this thesis, the EMD is modeled as an input delay in the musculoskeletal dynamics.

However, controllers mentioned above are all designed for unidirectional limb movements and can only deliver current through a single channel; when extending the lower leg through NMES, only quadriceps muscles were stimulated through a single NMES channel. During the flexing period, the leg is lowered by a controlled reduction in currents to the quadriceps muscle, and the gravity of the shank to return to its original position. However, not all the situations can make use of the gravity, in a standing position or during a gait cycle, producing knee flexion and extension would require controlled stimulation of both hamstring and quadriceps (antagonistic pair), these actions are common in real life such as when kicking football a smooth and continuous swing movement of the lower limb will be required. Similarly, using NMES to achieve upper-limb movements in horizontal plane such elbow or wrist flexion and extension would need the participation of antagonistic pairs (e.g., biceps and triceps muscles). The need for multi-muscle control for advanced NMES applications has driven the developments of multi-channel controllers.

In recent years, iterative learning control (ILC) was used for FES applications for upper limbs [22-25]. In these studies, the stimulation was applied to triceps and anterior-deltoids muscles. However, the closed-loop control of the stimulation allowed only unidirectional joint movement like the flexion or extension of the muscles, movements in another direction relies on the robot arm that was attached to the patient's upper limb. This implies the controller may not be capable of controlling antagonistic muscles to flex as well as extend an upper-limb joint in a 
smooth and relative more efficient way. Also, no modeling or stability analysis was included in it to prove its stability mathematically.

Downey et al. [9], purposed an asynchronous NMES controller for the multiple muscles in the quadriceps muscle group was delivered through four-channels. Its purpose was to reduce muscle fatigue caused by high-frequency stimulation when using single channel. The switching between the channels is based on a fixed time periodic signal and the switching time window can be tuned arbitrarily small by increasing the gain. In a more recent work, Downey et al. [48] improved the previous controller by introducing a switched systems analysis to ensure the controller to switch between different channels instantaneously without a transition period. The switching time window is not preferred in the real application as it made stimulation channels overlap and cause muscle fatigue. But these controllers' objective was to reduce fatigue by delivering stimulation between different channels periodically rather to achieve multi-directional movement control of antagonist muscle groups. They can only be applied to uni-directional NMES-induced movements because switching scheme was only proposed for agonist muscles.

Because the area of multi-channel control of antagonist muscle pairs is relatively untouched, the goal of this thesis is to design an antagonist muscles controller with a delay compensator to fulfill the need for advanced NMES tasks. 


\subsection{CONTROLLER DEVELOPMENT}

In this chapter, the musculoskeletal system model is introduced, and the closed-loop tracking controller for dual control of NMES tasks such as limb extension and flexion is presented. The objective is to achieve smooth and continuous limb extension and flexion movements.

\subsection{MUSCULOSKELETAL SYSTEM}

In order to develop the controller, the first step is to define the musculoskeletal model for the design. The uncertain nonlinear musculoskeletal dynamics are modeled similar to [7] and are defined as

$$
M_{I}+M_{e}+M_{g}+M_{v}+d=T_{1}(t-\tau)-T_{2}(t-\tau),
$$

where $M_{I} \in \mathbb{R}$ denotes the inertial force about the joint, $M_{e} \in \mathbb{R}$ denotes the elastic effects due to joint stiffness, $M_{g} \in \mathbb{R}$ denotes the gravitational forces and $M_{v} \in \mathbb{R}$ denotes the viscous effects from damping in the musculoskeletal system. In (3.1), $d \in \mathbb{R}$ represents any bounded unknown disturbance and/or unmodeled dynamics, and $T_{1} \in \mathbb{R}$ denotes torque produced for extension while $T_{2} \in \mathbb{R}$ denotes torque produced for flexion. 
The inertial effects in (3.1) are modeled as

$$
M_{I} \triangleq J \ddot{q}
$$

where $J \in \mathbb{R}^{+}$is the moment of inertia of the limb.

The elastic effects due to joint stiffness are modeled as

$$
M_{e} \triangleq k_{1}\left(\exp \left(-k_{2} q\right)\right)\left(q-k_{3}\right)
$$

where $k_{1}, k_{2}, k_{3} \in \mathbb{R}^{+}$are unknown parameters.

The gravitational and viscous effects are modeled as [6]

$$
M_{g} \triangleq m g l \sin (q), M_{v} \triangleq B_{1} \tanh \left(-B_{2} \dot{q}\right)+B_{3} \dot{q},
$$

where $m \in \mathbb{R}^{+}$is the unknown mass of the limb, $l \in \mathbb{R}^{+}$denotes the unknown distance from the joint to the lumped center of mass of the limb, and $q, \dot{q}, \ddot{q} \in \mathbb{R}$ are the angular position, velocity, and acceleration of the limb, respectively.

The torque produced for extension/flexion is related to musculotendon force that is generated by NMES and is defined as

$$
T_{i} \triangleq \varsigma_{i} F_{T, i}, i=1,2
$$

where $\varsigma_{i} \in \mathbb{R}$ is the positive moment arm for the corresponding muscle of the limb and $F_{T, i} \in \mathbb{R}$ is the musculotendon force generated by the stimulated muscle.

The musculotendon force $F_{T, i} \in \mathbb{R}$ in (3.5) is defined as

$$
F_{T, i} \triangleq \eta_{i} u_{i}, i=1,2
$$

where $\eta_{i} \in \mathbb{R}^{+}, i=1$, 2 denotes an unknown nonlinear function of the force-length/force-velocity relationship, and $u_{i} \in \mathbb{R}$ is the normalized applied voltage potential across the muscles.

The unknown functions in the active dynamics of the muscles are grouped in $\Omega_{i} \in \mathbb{R}$ as 


$$
\Omega_{i} \triangleq \varsigma_{i} \eta_{i}, i=1,2
$$

and can be bounded as

$$
\Omega_{i} \leq \zeta_{\Omega_{i}}, i=1,2
$$

where $\zeta_{\Omega_{i}} \in \mathbb{R}^{+}$is a constant.

Remark: In order to simplify the control design process, the muscle activation dynamics were not taken into account.

The following assumptions were made for the control development and stability analysis:

Assumption 1: Signals $q, \dot{q}$; which denote the generalized position and velocity are measurable.

Assumption 2: The nonlinear functions $\eta_{i}(q, \dot{q})$ and moment arm $\varsigma_{i}(q)$ are non-zero, positive, bounded functions, and their first time derivatives exist and are continuous and bounded based on the data. Thus $\Omega_{i}$ is also non-zero, positive bounded and its first time derivative exists, is bounded and continuous.

Assumption 3: The EMD, denoted by $\tau$, is assumed to be a known constant. Factors that may cause it to be a time-varying phenomenon such as fatigue are ignored. In addition, the EMDs in the opposing muscles are assumed to be equal.

Assumption 4: The desired trajectory and its time derivatives $q_{d}, \dot{q}_{d}, \ddot{q}_{d} \in \mathbf{R}$ are bounded and continuous.

Notation: A delayed state in the subsequent control development and analysis is denoted as $x(t-\tau)$ or as $x_{\tau}$ while a non-delayed state is denoted as $x(t)$ or as $x$. Any term, $X$, multiplied by the inverse of another term, $B$, is denoted as a subscript (i.e., $X_{B}$ ). 


\subsection{CONTROL DEVELOPMENT}

The control objective is to develop a tracking controller that could make the limb joint angle of the musculoskeletal dynamics in (3.1) track a desired trajectory, $q_{d} \in \mathbb{R}$. The position tracking error, $e_{1} \in \mathbb{R}$, and auxiliary tracking error, $e_{2} \in \mathbb{R}$, are defined as

$$
\begin{gathered}
e_{1} \triangleq q_{d}-q, \\
e_{2} \triangleq \dot{e}_{1}+\alpha e_{1}-\beta e_{z},
\end{gathered}
$$

where $\alpha, \beta \in \mathbb{R}^{+}$are control gains and the auxiliary signal, $e_{z} \in \mathbb{R}$, is defined as

$$
e_{z}=\int_{t-\tau}^{t} v(\theta) d \theta .
$$

After taking the time derivative of (3.10), then multiplying by the moment of inertia of a $\operatorname{limb} J$, and utilizing (3.1), (3.2), and(3.5), the open loop error dynamics is expressed as

$$
\begin{aligned}
J \dot{e}_{2}= & J \ddot{q}_{d}+M_{e}+M_{g}+M_{v}-\Omega_{1} u_{1 \tau} \\
& +\Omega_{2} u_{2 \tau}+d+J \alpha \dot{e}_{1}-J \beta\left(v-v_{\tau}\right) .
\end{aligned}
$$

To smoothly transition between stimulation of the flexor and extensor muscles, two switching signals, $S_{1}, S_{2} \in \mathbb{R}^{+}$, are defined as

$$
\begin{aligned}
& S_{1}=\frac{1+\tanh \left(\kappa e_{1}\right)}{2}, \\
& S_{2}=\frac{1-\tanh \left(\kappa e_{1}\right)}{2},
\end{aligned}
$$

and $\kappa \in \mathbb{R}^{+}$is a control gain that determines the transition rate. 
The normalized stimulation, $u_{i} \in \mathbb{R}^{+}$, can be expressed as

$$
\begin{aligned}
& u_{1}(t-\tau) \triangleq S_{1} \frac{v(t-\tau)-V_{t, 1}}{\Delta V_{1}}, \\
& u_{2}(t-\tau) \triangleq S_{2} \frac{v(t-\tau)-V_{t, 2}}{\Delta V_{2}},
\end{aligned}
$$

where $\Delta V_{i}=V_{s, i}-V_{t, i}, i=1,2$ and $V_{s, i} \in \mathbb{R}^{+}$is the saturation voltage which results in the maximum contraction and $V_{t, i} \in \mathbb{R}^{+}$is the threshold voltage which is the minimum voltage required to keep the $i$ th muscles in tension. The control input is denoted by $v$ and will be subsequently defined.

To facilitate the control development an auxiliary input gain function, $\Omega \in \mathbb{R}$, is defined as

$$
\Omega \triangleq\left[S_{1} \frac{\Omega_{1}}{\Delta V_{1}}-S_{2} \frac{\Omega_{2}}{\Delta V_{2}}\right]
$$

To avoid a singularity when $\Omega=0$, a constant $\delta \in \mathbb{R}^{+}$is added to $\Omega$ in the new auxiliary function, $\chi \in \mathbb{R}^{+}$defined as

$$
\chi=\Omega+\delta, \delta>|\Omega|,
$$

and can be bounded as

$$
\chi_{1} \leq|\chi| \leq \chi_{2}
$$

Assumption 5: The unknown disturbance $d(t)$ is bounded and its first and second derivatives with respect to time exist and are bounded, and based on assumption 1 and 2 the ratio $d(t) / \chi(q, \dot{q})$, is also bounded and its first and second time derivatives exists and are bounded.

Assumption 6: The ratio $J / \chi(q, \dot{q})$, denote as $J_{\chi}(q, \dot{q}) \in \mathbb{R}^{+}$, can be upper bounded as 


$$
J_{1}<\left|J_{\chi}\right|<J_{2} \text {, }
$$

where $J_{1}, J_{2} \in \mathbb{R}^{+}$are known constants.

To facilitate the subsequent stability analysis, the error between $\beta$ and $J_{\chi}^{-1}(q, \dot{q})$, is defined by

$$
\xi=\beta-\frac{\chi}{J},
$$

where $\xi(q, \dot{q}) \in \mathbb{R}^{+}$satisfies the following bound:

$$
|\xi| \leq \bar{\xi},
$$

and $\bar{\xi} \in \mathbb{R}^{+}$is a known constant.

Using (3.14) and (3.16), (3.12) is expressed as

$$
\begin{aligned}
J \dot{e}_{2}= & J \ddot{q}_{d}+M_{e}+M_{g}+M_{v}-\chi v_{\tau}+S_{1} \Omega_{1} \frac{V_{t, 1}}{\Delta V_{1}} \\
& -S_{2} \Omega_{2} \frac{V_{t, 2}}{\Delta V_{2}}+d+J \alpha \dot{e}_{1}-J \beta\left(v-v_{\tau}\right) .
\end{aligned}
$$

Dividing the open loop error system by $\chi$ and using (3.19) results in

$$
\begin{aligned}
& J_{\chi} \dot{e}_{2}=\quad J_{\chi} \ddot{q}_{d}+M_{e \chi}+M_{g \chi}+M_{v \chi}-v \\
& +\left(S_{1} \Omega_{1} \frac{V_{t, 1}}{\Delta V_{1}}-S_{2} \Omega_{2} \frac{V_{t, 2}}{\Delta V_{2}}+\delta v_{\tau}\right) \chi^{-1} \\
& +d+J \alpha \dot{e}_{1}-J \beta\left(v-v_{\tau}\right) .
\end{aligned}
$$

where

$$
M_{e \chi}=\frac{M_{e}}{\chi}, M_{g \chi}=\frac{M_{g}}{\chi}, M_{v \chi}=\frac{M_{v}}{\chi} .
$$

Based on the subsequent stability analysis, the control input $v \in \mathbb{R}$ is designed as

$$
v=K e_{2},
$$


where $K \in \mathbb{R}^{+}$is a known control gain that can be expanded as

$$
K=K_{1}+K_{2}+K_{3} \text {, }
$$

where $K_{1}, K_{2}$ and $K_{3} \in \mathbb{R}^{+}$are known constants.

After using the control input (3.24), the closed loop error system can be written as

$$
\begin{gathered}
J_{\chi} \dot{e}_{2}=\quad-\frac{1}{2} \dot{J}_{\chi} e_{2}+\bar{\psi}+\tilde{\psi}-e_{1} \\
-K e_{2}-K J_{\chi} \xi\left(e_{2}-e_{2 \tau}\right) \\
+\Pi+\delta v_{\tau} \chi^{-1},
\end{gathered}
$$

where

$$
\Pi=\left(S_{1} \Omega_{1} \frac{V_{t, 1}}{\Delta V_{1}}-S_{2} \Omega_{2} \frac{V_{t, 2}}{\Delta V_{2}}\right) \chi^{-1},
$$

and is bounded as

$$
\bar{\Pi} \triangleq|\Pi| \leqslant S_{1} V_{t, 1} \frac{\zeta_{\Omega_{1}}}{\Delta V_{1} \chi_{1}}+S_{2} V_{t, 2} \frac{\zeta_{\Omega_{2}}}{\Delta V_{2} \chi_{1}},
$$

and $\tilde{\psi}\left(e_{1}, e_{2}, t, \tau\right), \bar{\psi}\left(q, \dot{q}, q_{d}, \dot{q}_{d}, \ddot{q}_{d}, t\right) \in \mathbf{R}$ denote the following auxiliary signals

$$
\begin{gathered}
\psi \triangleq \frac{1}{2} \dot{J}_{\chi} e_{2}+J_{\chi} \ddot{q}_{d}+M_{e \chi}+M_{g \chi}+M_{v \chi} \\
+J_{\chi} \alpha \dot{e}_{1}+e_{1} \\
\psi_{d} \triangleq \quad \begin{array}{c}
J_{\chi}\left(q_{d}, \dot{q}_{d}\right) \ddot{q}_{d}+M_{e \chi}\left(q_{d}, \dot{q}_{d}\right) \\
+M_{g \chi}\left(q_{d}, \dot{q}_{d}\right)+M_{v \chi}\left(q_{d}, \dot{q}_{d}\right),
\end{array} \\
\tilde{\psi}=\psi-\psi_{d}, \quad \bar{\psi} \triangleq \psi_{d}+d_{\chi} .
\end{gathered}
$$

By applying the Mean Value Theorem, $\tilde{\psi}\left(e_{1}, e_{2}, t, \tau\right)$ can be upper bounded by statedependent terms as

$$
|\tilde{\psi}| \leq \rho(\|z\|)\|z\|,
$$


and $\rho(\|z\|) \in \mathbb{R}$ is a positive, globally invertible non-decreasing function and $z$ is defined as

$$
z(t) \triangleq\left[e_{1}, e_{2}, e_{z}\right]^{T}
$$

The second auxiliary signal, $\bar{\psi}\left(q, \dot{q}, q_{d}, \dot{q}_{d}, \ddot{q}_{d}, t\right)$, can be upper bounded as

$$
|\bar{\psi}| \leq \zeta_{S}
$$

where $\zeta_{s} \in \mathbb{R}^{+}$is a constant. Based on the subsequent stability analysis, LK functionals: $P(v, t, \tau) \in \mathbb{R}$ and $Q\left(e_{2}, t, \tau\right) \in \mathbb{R}$ are defined as

$$
\begin{gathered}
P=\omega \int_{t-\tau}^{t}\left(\int_{s}^{t} v(\theta)^{2} d \theta\right) d s, \\
Q=\frac{\left(\bar{\xi} J_{2} K+\frac{K \delta}{\chi_{1}}\right)}{2} \int_{t-\tau}^{t} e_{2}(\theta)^{2} d \theta,
\end{gathered}
$$

where $\omega \in \mathbb{R}^{+}$is a known constant. 


\subsection{STABILITY ANALYSIS}

Theorem 1. The controller given in (3.24) ensures semi-global uniformly ultimately bounded tracking

$$
|y(t)| \leq \epsilon_{0} \exp \left(-\epsilon_{1} t\right)+\epsilon_{2}
$$

where $\epsilon_{0}, \epsilon_{1}, \epsilon_{2} \in \mathbb{R}^{+}$denotes constants, provided the control gains $\alpha, \beta$ and $K$ introduced in (3.10) and (3.24) are selected according to the sufficient conditions

$$
\begin{gathered}
\alpha>\frac{\beta^{2} \gamma^{2}}{4}, \\
K_{3}>\omega K^{2} \tau+2 \bar{\xi} J_{2} K+\frac{K \delta}{\chi_{1}},
\end{gathered}
$$

where the known positive constants $\beta, \delta, \chi_{1}, J_{2}, K, \omega$ are defined in (3.10), (3.16), (3.17), (3.18), (3.24) and (3.35), $\tau$ is the input delay and $\gamma \in \mathbb{R}^{+}$is a subsequently defined constant.

Proof. Let $y(t) \in \mathcal{D} \subset \mathbb{R}^{4}$ be define as

$$
y \triangleq\left[e_{1}, e_{2}, \sqrt{P}, \sqrt{Q}\right]^{T}
$$

A positive definite Lyapunov functional candidate $V(y, t): \mathcal{D} \times[0 \infty) \rightarrow \mathbb{R}$ is defined as

$$
V \triangleq \frac{1}{2} e_{1}^{2}+\frac{1}{2} J_{\chi} e_{2}^{2}+P+Q
$$

and satisfies the following inequalities 


$$
\lambda_{1}\|y\|^{2} \leq V \leq \lambda_{2}\|y\|^{2},
$$

where $\lambda_{1}, \lambda_{2} \in \mathbb{R}^{+}$are known constants. Taking the time derivative of (4.4), using the Leibniz integral rule to differentiate $P$ and $Q$, substituting (3.10) and (3.26), and canceling out like terms results in

$$
\begin{array}{rcc}
\dot{V}= & -\alpha_{1} e_{1}^{2}-K e_{2}^{2}+\beta e_{z} e_{1}+\omega \tau v^{2} \\
& +e_{2}\left[\bar{\psi}+\tilde{\psi}-K J_{\chi} \xi\left(e_{2}-e_{2 \tau}\right)+\Pi+\delta v_{\tau} \chi^{-1}\right] \\
& +\frac{\left(\bar{\xi} J_{2} K+\frac{K \delta}{\chi_{1}}\right)}{2}\left(e_{2}^{2}-e_{2 \tau}^{2}\right)-\omega \int_{t-\tau}^{t} v(\theta)^{2} d \theta .
\end{array}
$$

After using (3.8), (3.17), (3.18), (3.20), (3.28), (3.32) and (3.34), (4.6) can be upper bounded as

$$
\begin{gathered}
\dot{V} \leq \quad-\alpha e_{1}^{2}-\left(K-\bar{\xi} J_{2} K\right) e_{2}^{2}+\omega \tau v^{2}+\left|e_{2}\right| \zeta_{s}+\left|e_{2}\right| \bar{\Pi} \\
+\left|e_{2}\right| \rho(\|z\|)\|z\|+\beta\left|e_{1}\left\|e_{z}\left|+\left(\bar{\xi} J_{2} K+\frac{K \delta}{\chi_{1}}\right)\right| e_{2 \tau}\right\| e_{2}\right| \\
+\frac{\left(\bar{\xi} J_{2} K+\frac{K \delta}{\chi_{1}}\right)}{2}\left(e_{2}^{2}-e_{2 \tau}^{2}\right)-\omega \int_{t-\tau}^{t} v(\theta)^{2} d \theta .
\end{gathered}
$$

Applying Young's Inequality the following terms in (4.7) can be bounded as

$$
\begin{gathered}
\beta\left|e_{1}\right|\left|e_{z}\right| \leq \frac{\beta^{2} \gamma^{2}}{4} e_{1}^{2}+\frac{1}{\gamma^{2}} e_{z}^{2}, \\
\left|e_{2 \tau}\right|\left|e_{2}\right| \leq \frac{1}{2} e_{2 \tau}^{2}+\frac{1}{2} e_{2}^{2},
\end{gathered}
$$

where $\gamma \in \mathbb{R}^{+}$is a known constant that is selected as

$$
\gamma>\sqrt{\frac{2 \tau}{\omega}} .
$$


Further, by using the Cauchy Schwarz inequality, the following term in (4.8) can be bounded as

$$
e_{z}^{2} \leq \tau \int_{t-\tau}^{t} v(\theta)^{2} d \theta
$$

After adding and subtracting $\frac{\tau}{\gamma^{2}} \int_{t-\tau}^{t} v(\theta)^{2} \mathrm{~d} \theta$ to (4.7) and utilizing (3.24), (3.25) and (4.11), (4.7) can be expressed as

$$
\begin{aligned}
\dot{V} \leq-\left(\alpha-\frac{\beta^{2} \gamma^{2}}{4}\right) e_{1}^{2}-\left(K-\omega K^{2} \tau-2 \bar{\xi} J_{2} K-\frac{K \delta}{\chi_{1}}\right) e_{2}^{2} \\
-\frac{1}{\tau}\left(\omega-\frac{2 \tau}{\gamma^{2}}\right) e_{z}^{2}+\left|e_{2}\right| \rho(\|z\|)\|z\| \\
+\left(\zeta_{S}+\bar{\Pi}\right)\left|e_{2}\right|-\frac{\tau}{\gamma^{2}} \int_{t-\tau}^{t} v(\theta)^{2} d \theta
\end{aligned}
$$

By using (3.25) and completing the squares, the inequality in (4.12) can be further upper bounded as

$$
\begin{gathered}
\dot{V} \leq-\left\{\Lambda-\frac{\rho^{2}(\|z\|)}{4 K_{1}}\right\}\|z\|^{2}-\frac{\tau}{\gamma^{2}} \int_{t-\tau}^{t} v(\theta)^{2} d \theta \\
+\frac{\left(\zeta_{S}+\bar{\Pi}\right)^{2}}{4 K_{2}},
\end{gathered}
$$

where

$$
\begin{gathered}
\Lambda \triangleq \min \left[\alpha-\frac{\beta^{2} \gamma^{2}}{4}, K_{3}-\omega K^{2} \tau-2 \bar{\xi} J_{2} K-\frac{K \delta}{\chi_{1}},\right. \\
\left.\frac{1}{\tau}\left(\omega-\frac{2 \tau}{\gamma^{2}}\right)\right] .
\end{gathered}
$$

Since

$$
\begin{array}{r}
\int_{t-\tau}^{t}\left(\int_{s}^{t} v(\theta)^{2} d \theta\right) d s \quad \leq \tau \sup _{s \in[t, t-\tau]}\left[\int_{s}^{t} v(\theta)^{2} d \theta\right] \\
=\tau \int_{s}^{t} v(\theta)^{2} d \theta
\end{array}
$$


and after utilizing (3.35), (3.36), and (4.3) the inequality in (4.13) can be rewritten as

$$
\begin{gathered}
\dot{V} \leq-\left(\Lambda-\frac{\rho^{2}(\|z\|)}{4 K_{1}}\right)\|z\|^{2}-\frac{K^{2} \tau}{\gamma^{2}\left(\bar{\xi}_{J_{2}} K+\frac{K \delta}{\chi_{1}}\right)} Q \\
-\frac{1}{2 \omega \gamma^{2}} P+\frac{\left(\zeta_{S}+\bar{\Pi}\right)^{2}}{4 K_{2}} .
\end{gathered}
$$

Using the definition of $z(t)$ in (3.33) and $y(t)$ in (4.3), the expression in (4.16) can be upper bounded as

$$
\dot{V} \leq-\Upsilon\|y\|^{2}-\left(\Lambda-\frac{\rho^{2}(\|z\|)}{4 K_{1}}\right)\left\|e_{z}\right\|^{2}+\frac{\left(\zeta_{s}+\bar{\Pi}\right)^{2}}{4 K_{2}},
$$

where $\Upsilon(\|z\|) \in \mathbb{R}^{+}$is

$$
\sigma \leq \Upsilon \triangleq \min \left[\Lambda-\frac{\rho^{2}(\|z\|)}{4 K_{1}}, \frac{K^{2} \tau}{\gamma^{2}\left(\bar{\xi} J_{2} K+\frac{K \delta}{\chi_{1}}\right)}, \frac{1}{2 \omega \gamma^{2}}\right]
$$

for some $\sigma \in \mathbb{R}^{+}$.

By further utilizing (4.5), the inequality in (4.17) can be expressed as

$$
\dot{V} \leq-\frac{\sigma}{\lambda_{2}} V+\frac{\left(\zeta_{S}+\bar{\Pi}\right)^{2}}{4 K_{2}} .
$$

Consider a set $S$ defined as

$$
\begin{aligned}
& S \triangleq \quad\left\{y(t) \in R^{4}\|y(0)\|<\right. \\
& \sqrt{\frac{\lambda_{1}}{\lambda_{2}} \min \left\{1, \frac{2 K \tau}{\left(\bar{\xi} J_{2}+\delta \chi_{1}^{-1}\right)}\right\} \rho^{-2}\left(2 \sqrt{\Lambda K_{1}}\right)-W},
\end{aligned}
$$




$$
W=\frac{\left(\zeta_{s}+\bar{\Pi}\right)^{2}}{4 K_{2} \sigma}
$$

The linear differential equation in (4.19) can be solved as

$$
V \leq V(0) e^{-\frac{\sigma}{\lambda_{2}} t}+\frac{\left(\zeta_{S}+\bar{\Pi}\right)^{2} \lambda_{2}}{4 K_{2} \sigma}\left[1-e^{-\frac{\sigma}{\lambda_{2}} t}\right]
$$

provided the control gains $\alpha$ and $K$ are selected according to the sufficient conditions in (4.2). The result in (4.1) can now be obtained from (4.21). Based on the definition of $y(\mathrm{t})$, the result in (4.21) indicates that $e_{1}(t), e_{2}(t) \in \mathcal{L}_{\infty}$ in $S$. Given that $e_{1}(t), e_{2}(t), q_{d}(t), \dot{q}_{d}(t) \in \mathcal{L}_{\infty}$ in $S$, (3.9), (3.10), and (3.24) indicates that $q(t), \dot{q}(t), v(t) \in \mathcal{L}_{\infty}$ in $S$. 


\subsection{SIMULATIONS \& EXPERIMENTS}

In order to test the performance of the designed controller in the previous chapters, simulations were conducted and followed by experiments on a testbed composed of electric motors to simulate antagonist muscle pairs. In the following section, the procedure and the results of the simulations and experiments are presented.

\subsection{SIMULATIONS}

In the SIMULINK simulation, a general single joint one-degree of freedom musculoskeletal system in the horizontal plane, (i.e., gravity cannot drive the limb in the opposite direction), was considered for testing the performance of this controller. The delay values were chosen as 80ms for both the flexor and extensor muscles and was added in the muscle model. The Simulink block diagram for the controller is shown in Appendix A.

The simulation results including the trajectory, stimulation output, error, and control input plot are shown in Figure 2 and Figure 3. The root mean squared error (RSME) for tracking the desired trajectory was calculated to be $2.58^{\circ}$. 

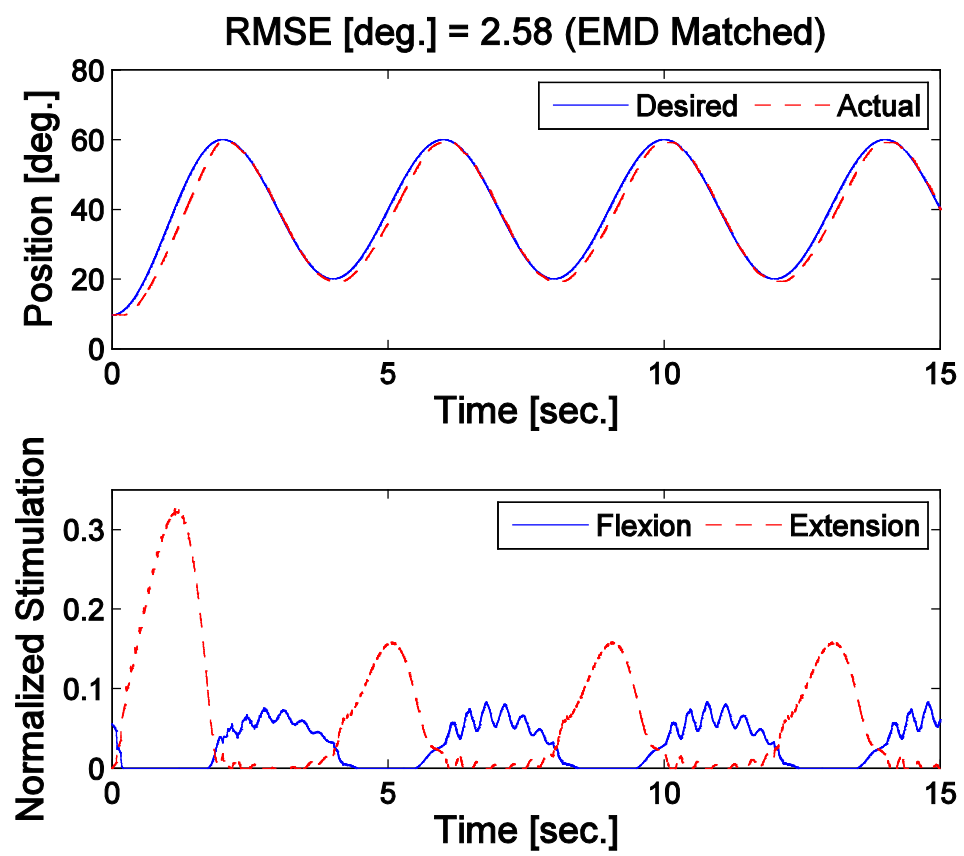

Figure 2. Trajectory and Normalized Stimulation $(\mathrm{u} 1, \mathrm{u} 2)$ Plots from Simulation
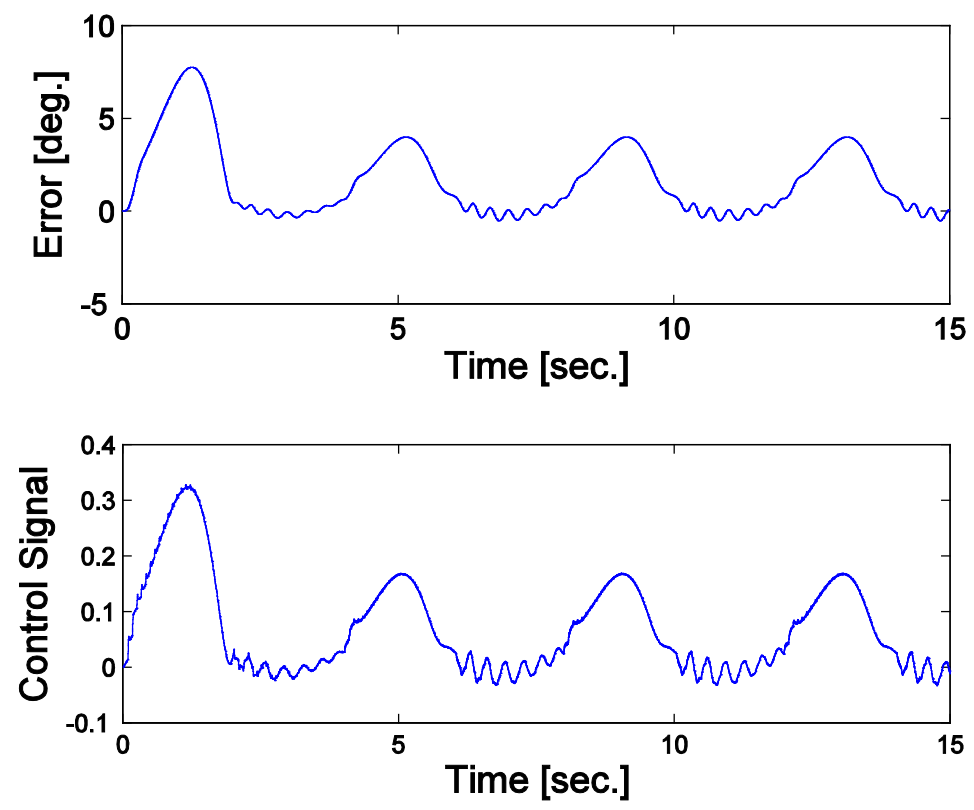

Figure 3. Normalized Error and Control Input ( v ) Plots from Simulation 
From the simulation results, it can be observed that despite the effects of the EMD, the controller is capable of driving the single joint musculoskeletal system in both directions (flexion and extension) in a smooth manner and maintain tracking to the desired trajectory.

Also, from the normalized stimulation plot, it can be observed that when switching occurs (transition between flexion and extension), there is a slight overlap causing cocontractions of antagonistic muscles. During the flexion period, the co-contraction continue to exist due to the oscillation caused by time delay, the activation of the extension muscle helped the limb to follow the desired trajectory with overshoot. It can also be seen that the extension stimulation magnitude is higher than the flexion, this is due to the different muscle parameters used for the flexor and extensor muscles in the musculoskeletal system.

During the process of the stability analysis, we assumed that EMD is known and has a precise number. During NMES application, EMD values can vary related to the muscle contraction type, characteristics of the subject such as gender, age, and the joint angle that the contraction needs to achieve. Previous research done by [43] reports that when receiving external electrical stimulation, the EMD could be ranging from $7 \mathrm{~ms}$ to $20 \mathrm{~ms}$. In [18], the EMD is measured to be ranging from $80 \mathrm{~ms}$ to $110 \mathrm{~ms}$. The variation of EMD values can be altered not only by the factors mentioned above but also can cause by methodological differences in experimental and measurement methods.

To examine the robustness to the variations in EMD values, the value of EMD in the delay compensation component of the controller were set to 75ms and 85ms whereas the EMD in the musculoskeletal system remained as 80ms. The results are shown in Figure 4 - Figure 7 below. 

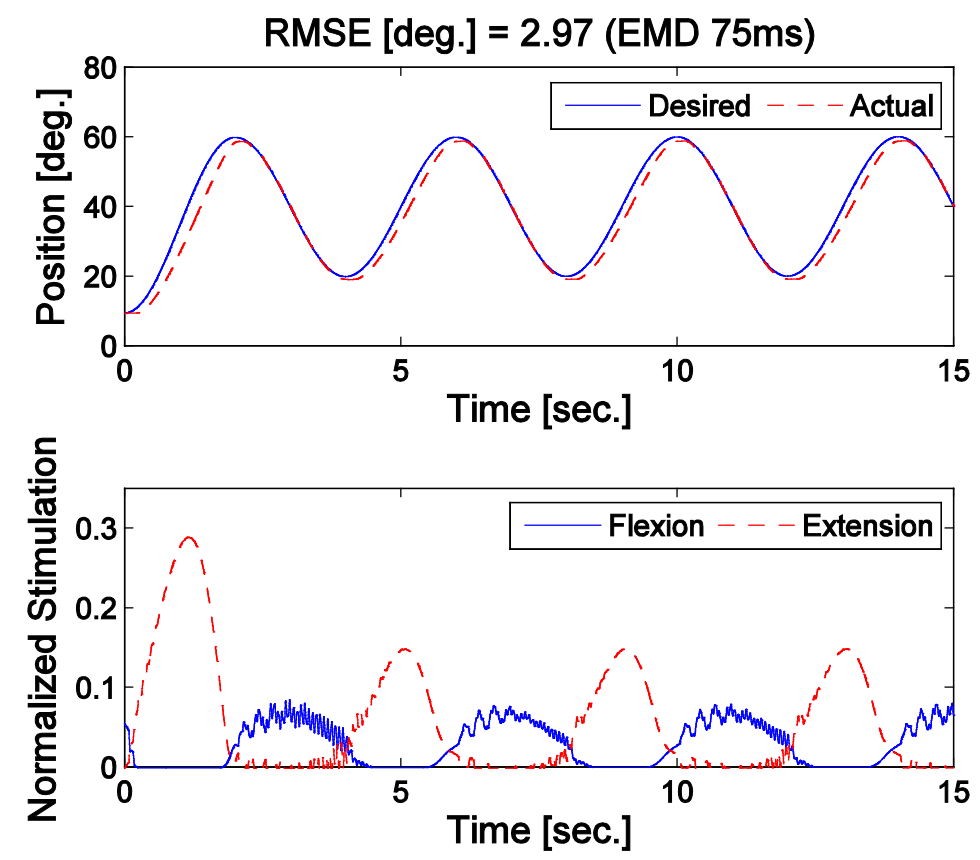

Figure 4. Trajectory and Normalized Stimulation (u1,u2) Plot from Simulation (75ms)
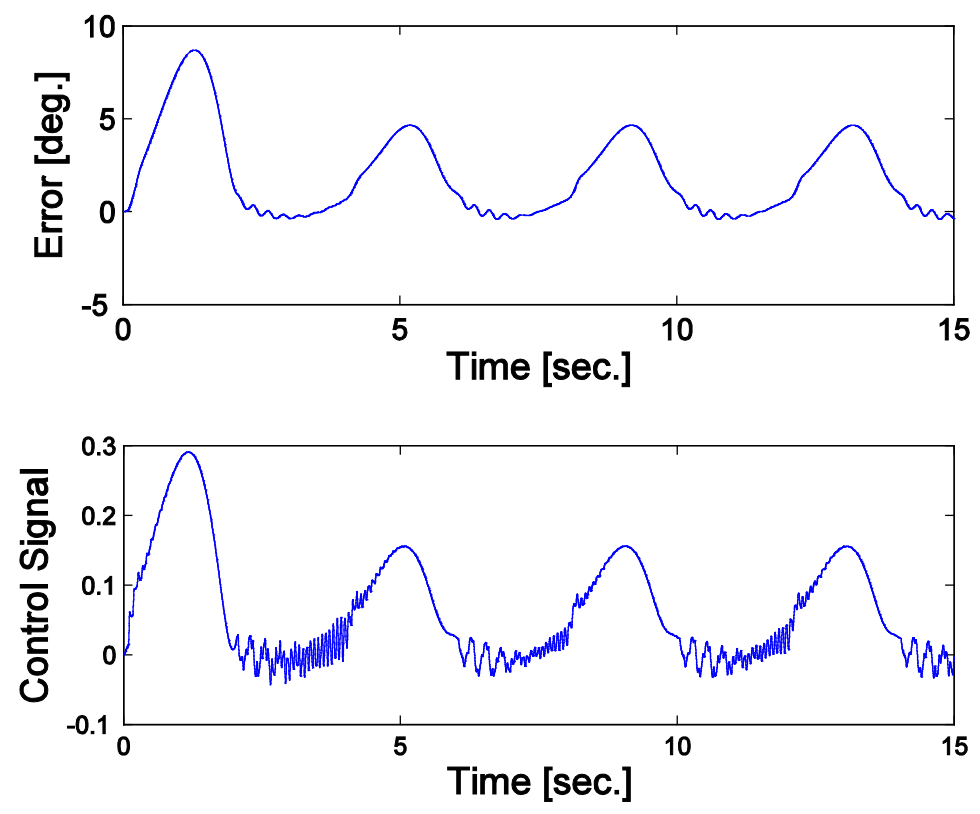

Figure 5. Normalized Error and Control Input ( v ) Plot from Simulation (75ms) 
Above is the simulation results when the value of EMD in the delay compensation component is set to $75 \mathrm{~ms}$. The RMSE is slightly increased compared to the previous simulation when the EMD value is matched between musculoskeletal system and delay compensator.

The results of simulation when the EMD value was set to 85ms are shown in Figure 6 and Figure 7.
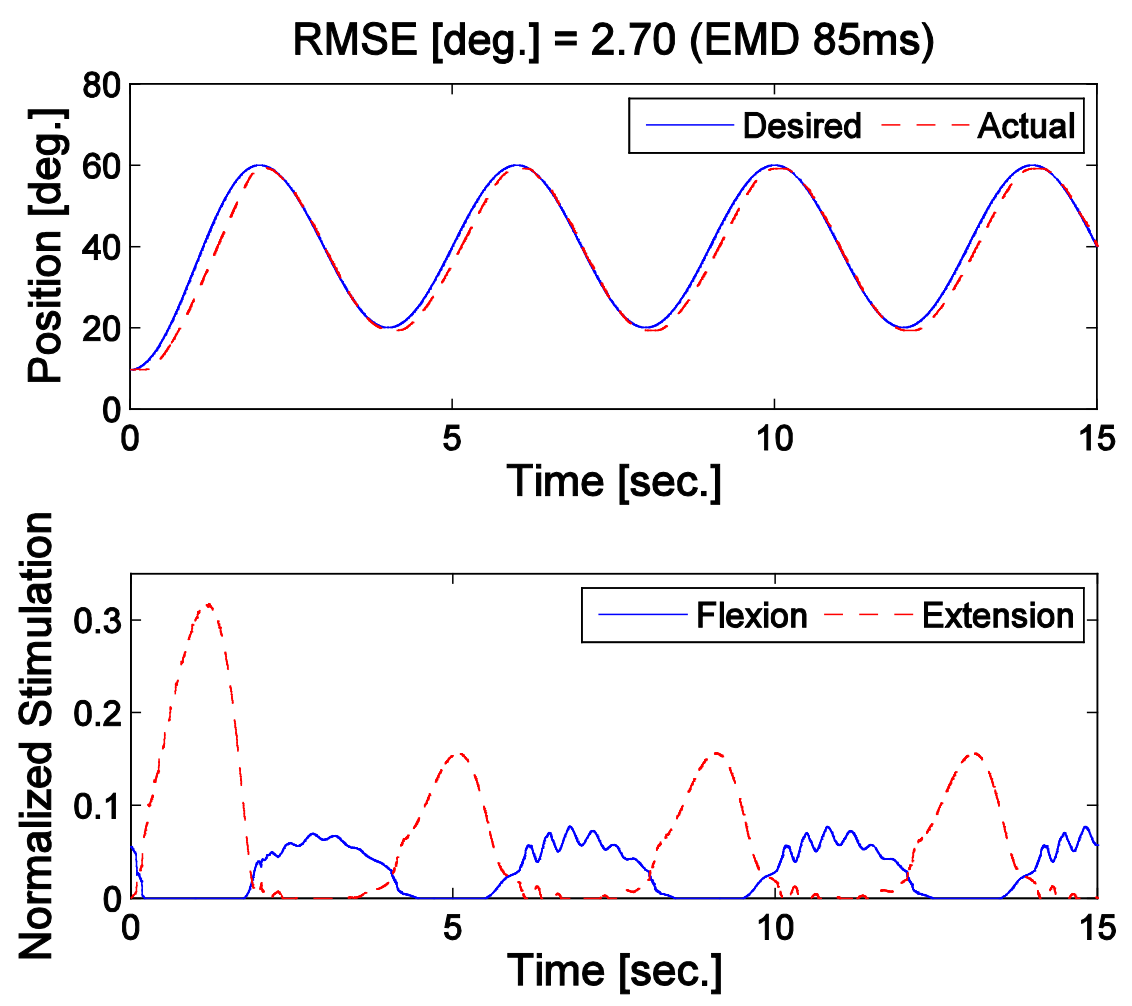

Figure 6. Trajectory and Normalized Stimulation Plot (u1,u2) from Simulation (85ms) 

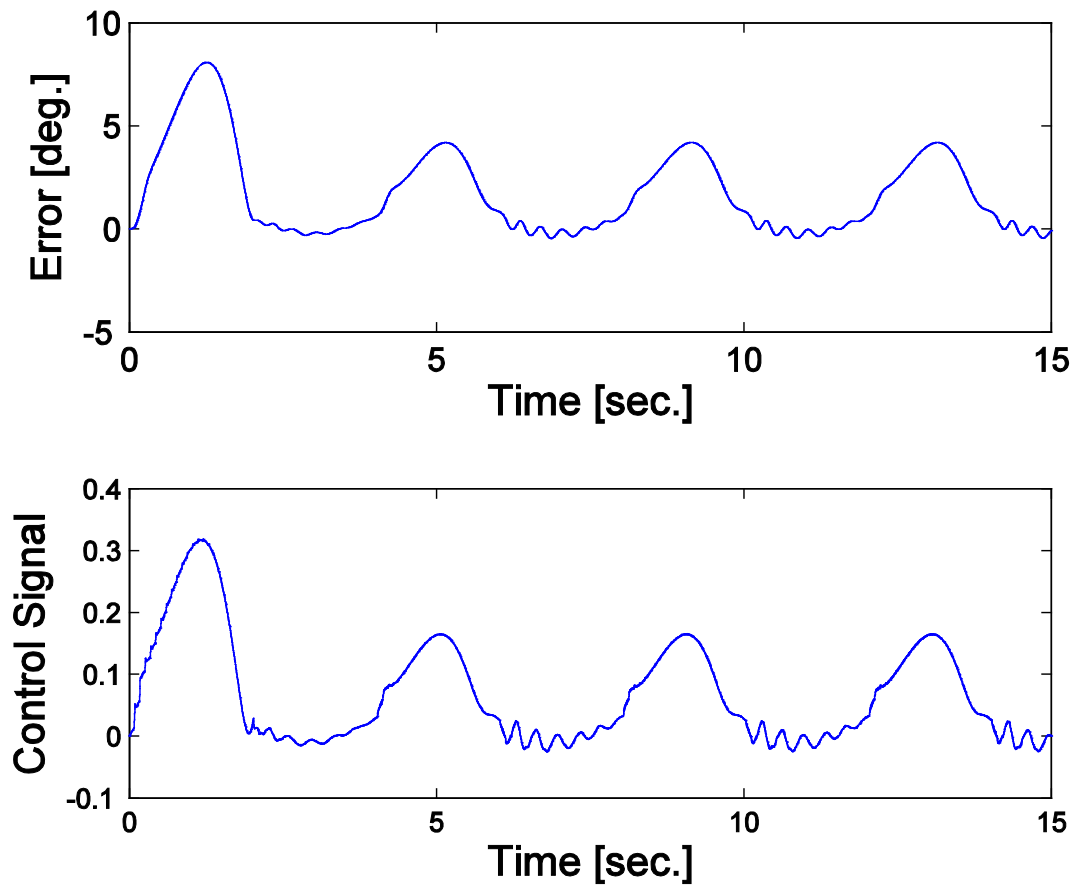

Figure 7. Normalized Error and Control Input ( v ) Plot from Simulation (85ms)

Table 1. RMSE of Simulation with Different EMD Value

\begin{tabular}{|c|c|}
\hline Estimated EMD Value (ms) & RMSE (deg.) \\
\hline 80 (Matched) & 2.58 \\
\hline 75 & 2.97 \\
\hline 85 & 2.70 \\
\hline
\end{tabular}

Table 1 above provides RMSE comparison between different EMD values used in the delay compensator. From the data above it can be observed that when the EMD value is drifting from the system's exact delay value, the RMSE of trajectory tracking increased slightly (0.1 0.4deg). More oscillation appeared during the stimulation when estimated EMD is different from actual EMD and co-contraction appeared during flexion period, This can be caused by the 
derivative part of the controller and variation of EMD value between delay compensator and musculoskeletal system. Overall, the system maintains stability and the tracking performance is still acceptable, which demonstrates the robustness of the controller.

\subsection{EXPERIMENTS}

An alternative approach was used for conducting the experiments before testing on human subjects. The testbed used an electric motors set-up to simulate the extensor and flexor muscle of a human musculoskeletal system. In the first part of the experiments, a single motor was used to test the performance of the controller. Later two motors were used and were combined to move in opposite direction to represent the extensor muscle and flexor muscle.

LPA-17 compact precision servo motors were used in the experiments. The motor has embedded encoders and can withstand high moment and axial loads due to the pre-loaded double row angular contact bearing. These motors were combined with DEP-090-18 digital servo drive to provide precise velocity control of the motor. Control input from the controller was given to the motor drive and based on the control input, the drive then produces amplified voltage to drive the motor. Encoder signal was buffered in the drive and transmitted to the controller.

\subsubsection{Single Motor Experiment}

The first part of the experiment was to use a single motor to represent both the flexor muscle and extensor muscle; the controller provided control output to the motor drive and motor to make it follow the desired trajectory. 
The SIMULINK block diagram is shown in Appendix A. A time delay was added to the system to represent EMD, and was set to $80 \mathrm{~ms}$. The experiment results are shown in Figure 8 and Figure 9.
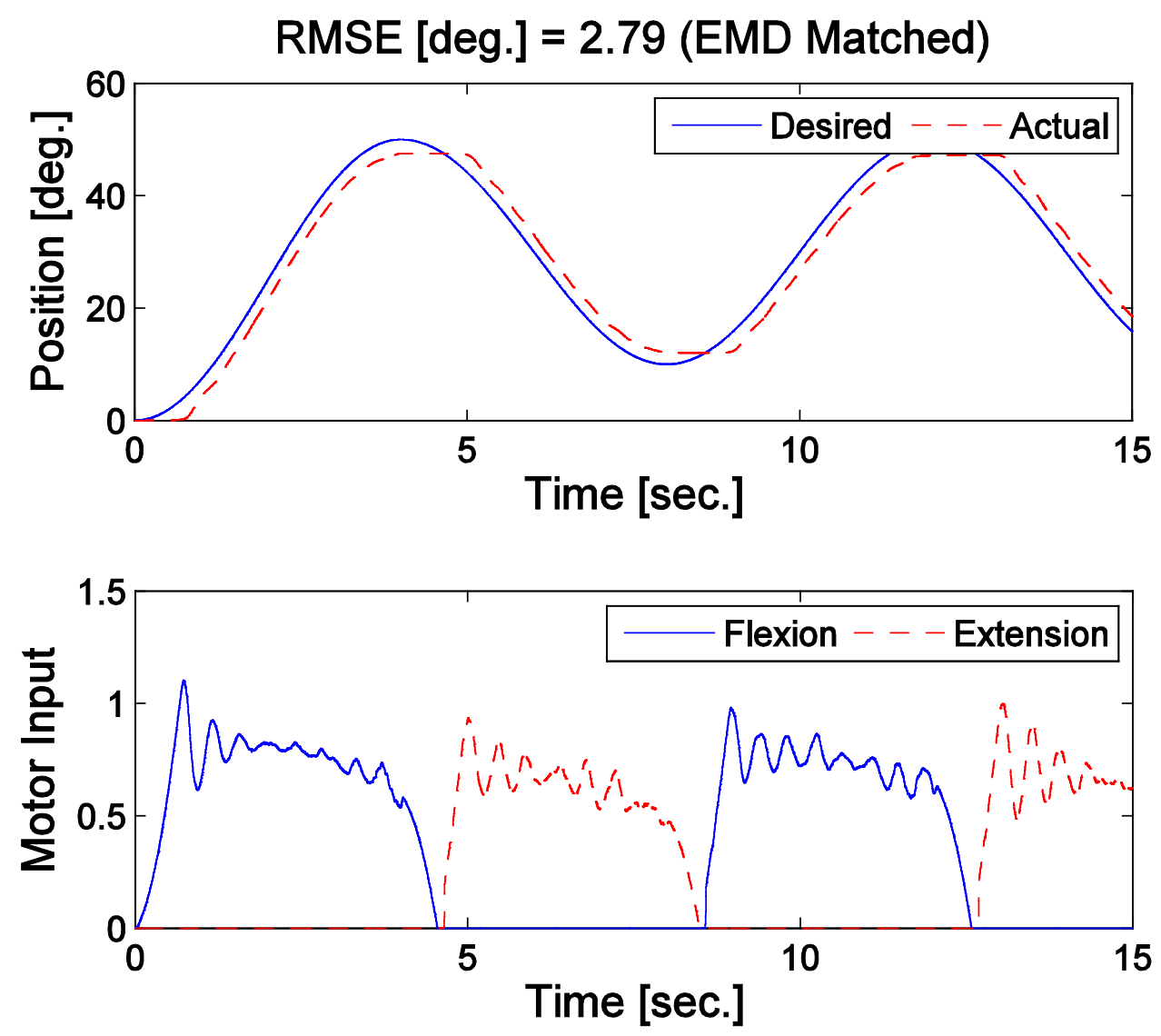

Figure 8. Trajectory and Motor Input (u1,u2) Plot from Single Motor Experiment 

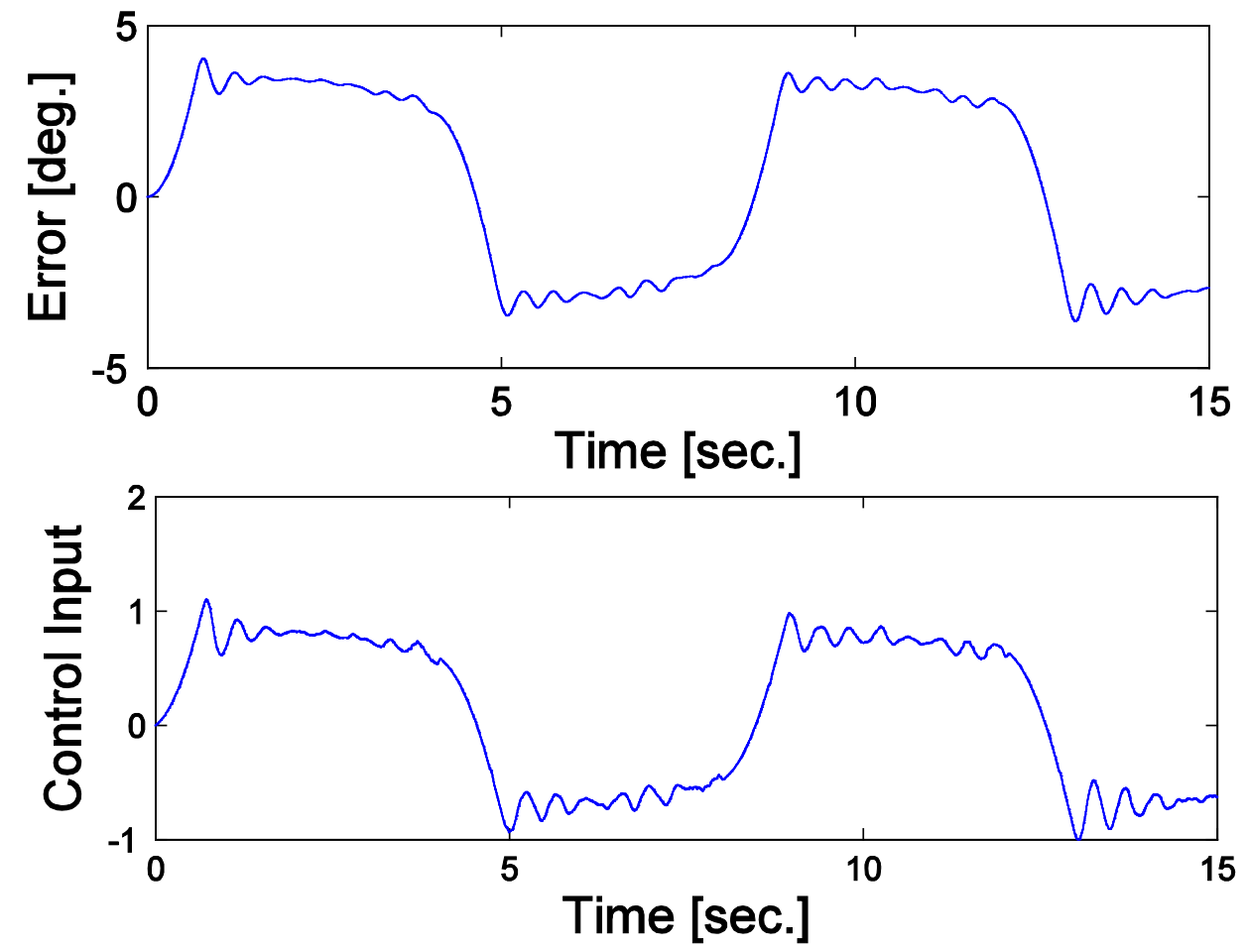

Figure 9. Error and Control Input Plot ( v ) from Single Motor Experiment

The root mean squared error (RSME) of tracking the desired trajectory was calculated to be $2.79^{\circ}$, which is slightly higher than the results from simulation. By increasing the gain $\kappa$ in the hyperbolic tangent function in (3.13), the switching time window became relatively short as observed in the figures above.

Similar to the simulation, the EMD value was set to $80 \pm 5 \mathrm{~ms}$ to examine the robustness of the controller. 

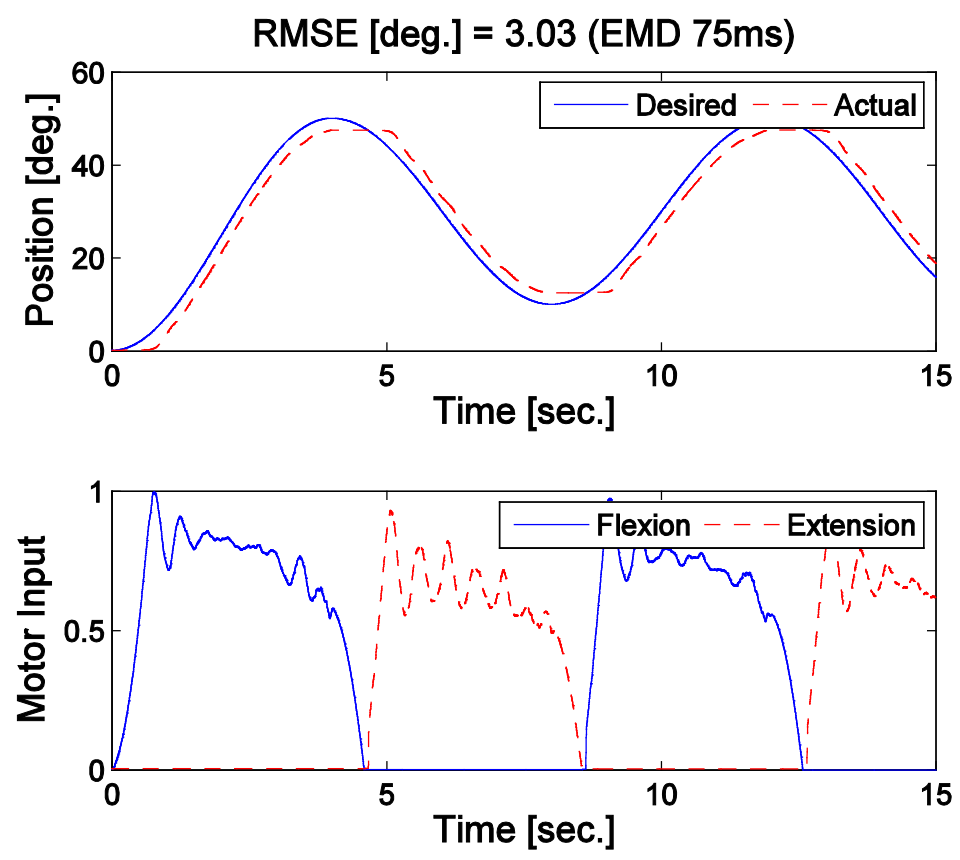

Figure 10. Trajectory and Motor Input (u1,u2) Plot from Single Motor Experiment (75ms)
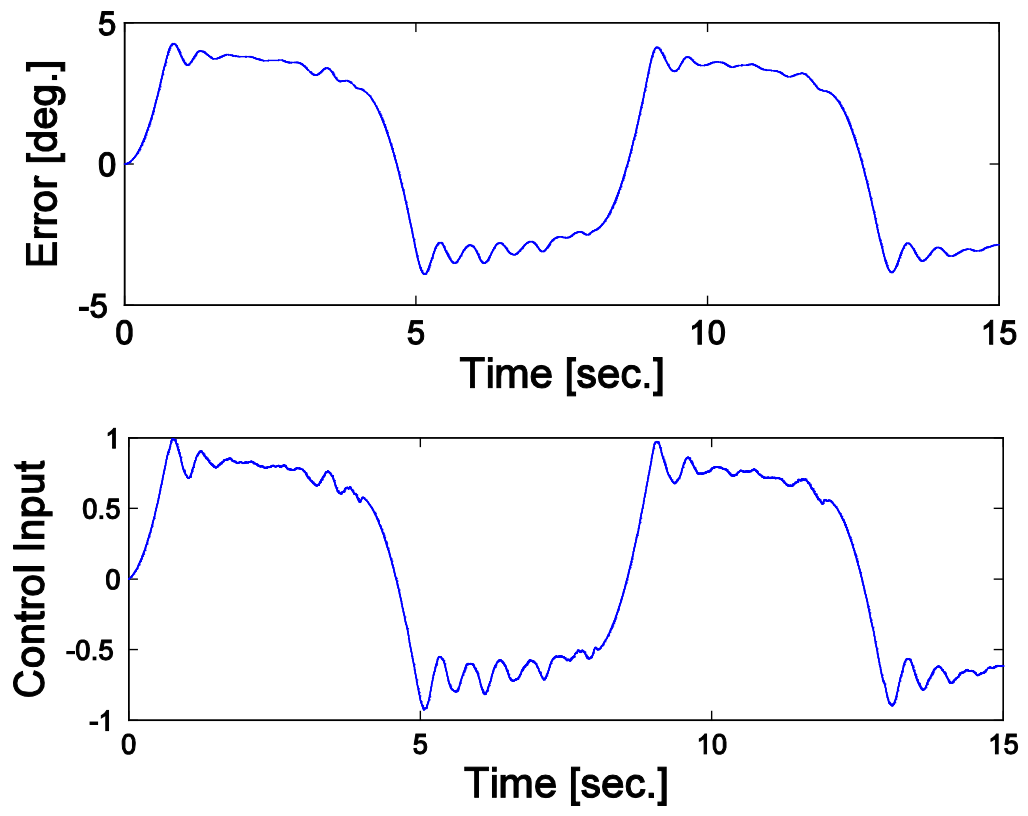

Figure 11. Error and Control Input Plot ( v ) from Single Motor Experiment (75ms) 

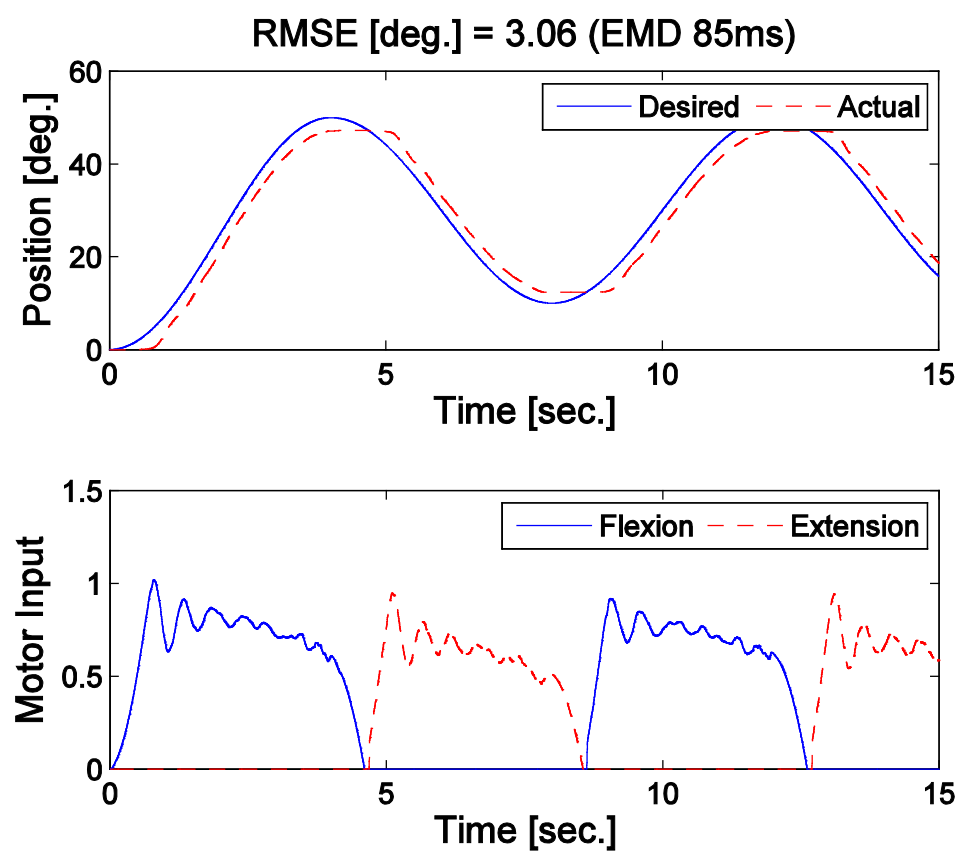

Figure 12. Trajectory and Motor Input (u1,u2) Plot from Single Motor Experiment (85ms)
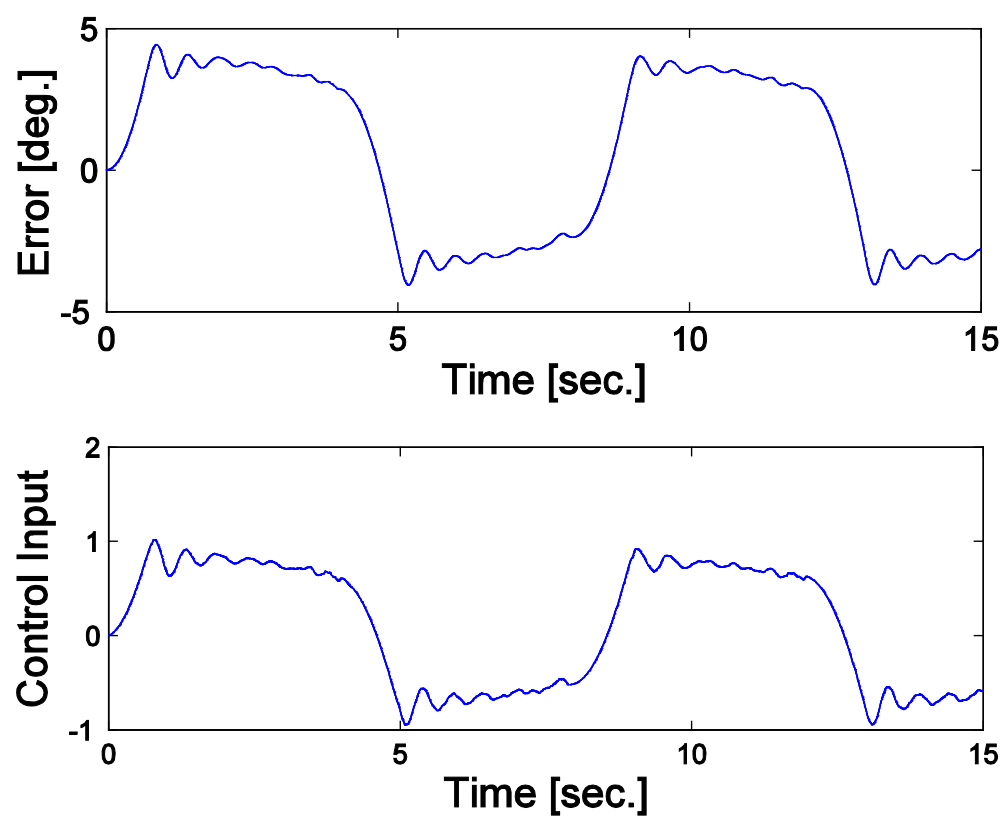

Figure 13. Error and Control Input Plot ( v ) from Single Motor Experiment (85ms) 
Table 2. RMSE of Single Motor Experiments with Different EMD Value

\begin{tabular}{|c|c|}
\hline Estimated EMD Value (ms) & RMSE (deg.) \\
\hline 80 (Matched) & 2.79 \\
\hline 75 & 3.03 \\
\hline 85 & 3.06 \\
\hline
\end{tabular}

From results above, it can be observed that when the EMD value in the compensator was not matched exactly to the EMD in the system, the RMS error will increase slightly similar to the simulation. Oscillations can be observed in the motor input, error and controller output plot. The controller still has acceptable performance even when EMD value for the delay compensator was not estimated precisely.

To further simulate the human musculoskeletal system to examine the controller's performance, the next move was to set up dual motors experiments and use separate motor to represent flexor and extensor muscles.

\subsubsection{Dual Motors Experiment}

In order to obtain results closer to the experiments on the human subject, the following experiments utilized two identical motors that were used in the previous experiment and each of them to represent the flexor muscle or extensor muscle, they were linked together to simulate a general single joint one-degree of freedom musculoskeletal system. 


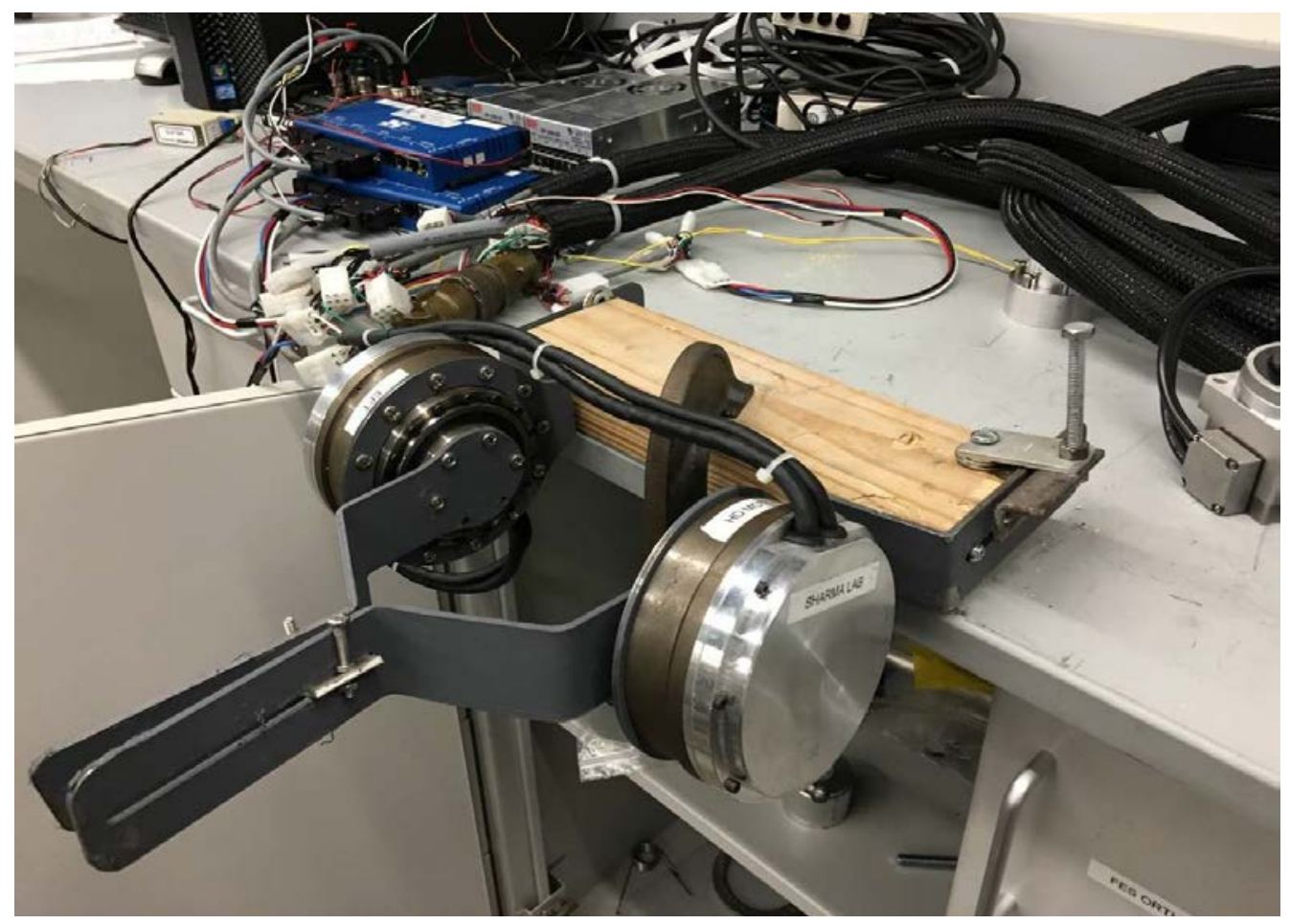

Figure 14. Dual Motors Experiment Testbed

Unlike the previous single motor experiment, in this experiment, each motor only moved in one direction and opposite to each other. When one motor moved towards one direction, no input was given to another motor and was dragged by the contralateral motor.

The experimental results are shown in Figure 15 and Figure 16. 

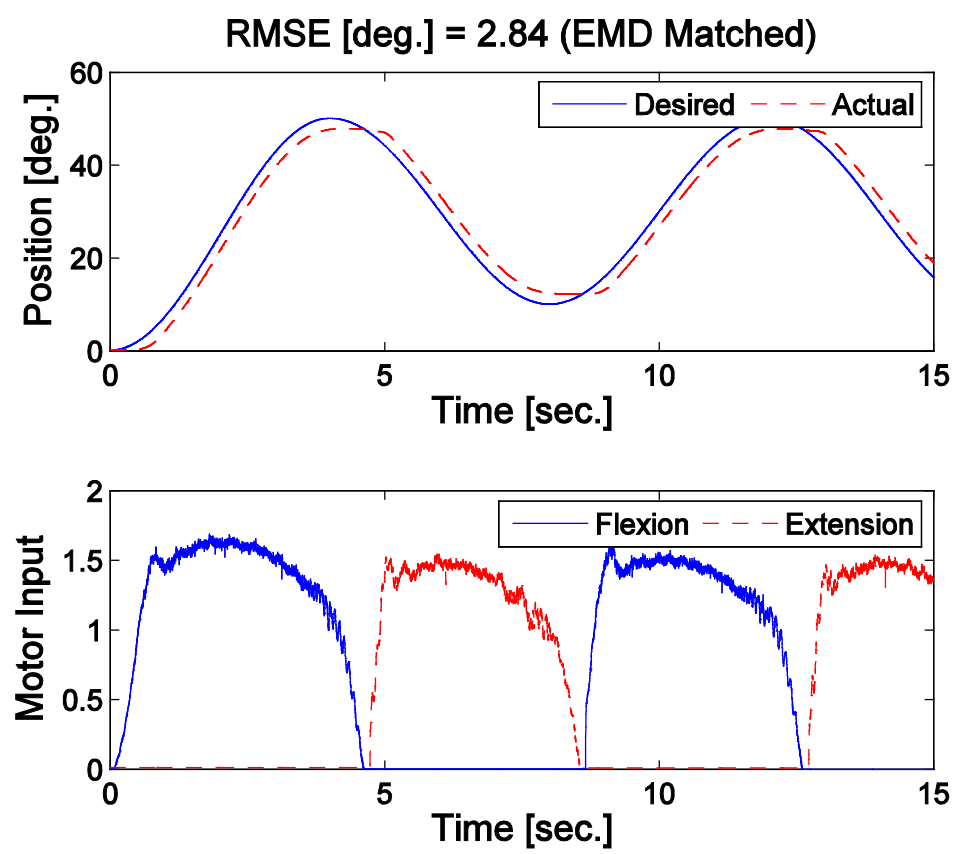

Figure 15. Trajectory and Motor Input (u1,u2) Plot from Dual Motors Experiment
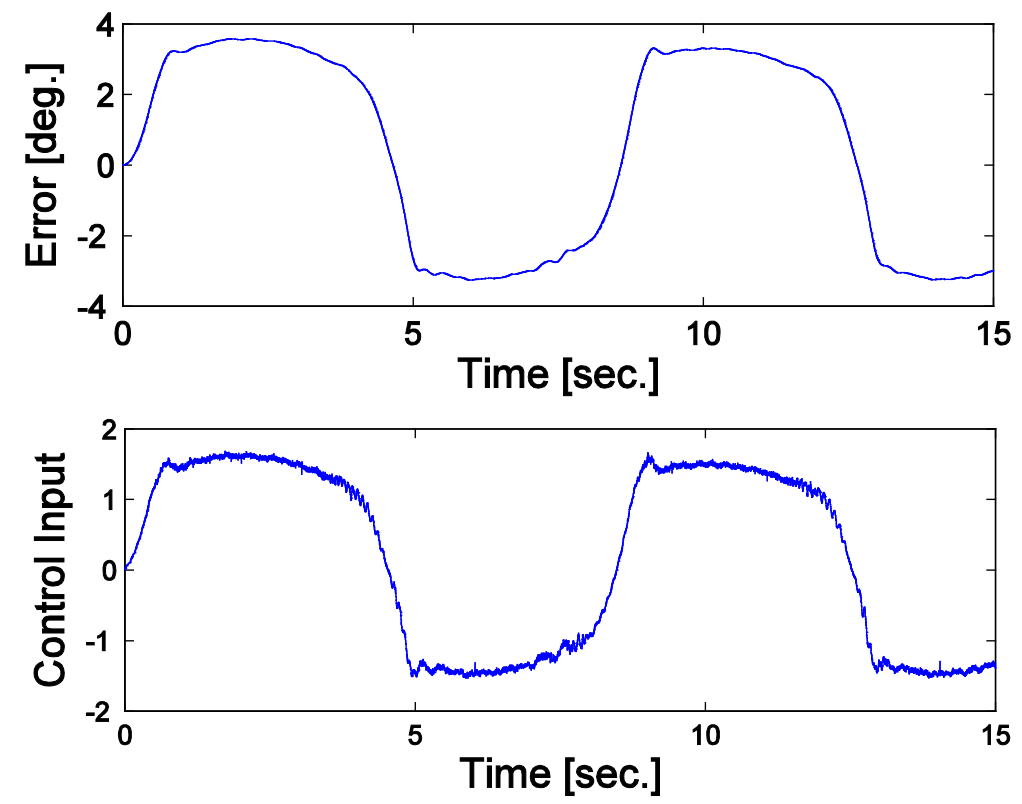

Figure 16. Error and Control Input ( v ) Plot from Dual Motors Experiment 
The RMSE of tracking for the dual motors experiment is $2.84^{\circ}$. It is larger than the experiment result from single motor. More oscillation occurs in the plots due to nonlinearity and disturbances, the difference in amplitude of the motor input is caused by the variations of the performance of each motor.

Again, the robustness of the controller was examined in dual motors situation, the EMD values were set to $80 \pm 5 \mathrm{~ms}$ while actual EMD remained at $80 \mathrm{~ms}$.
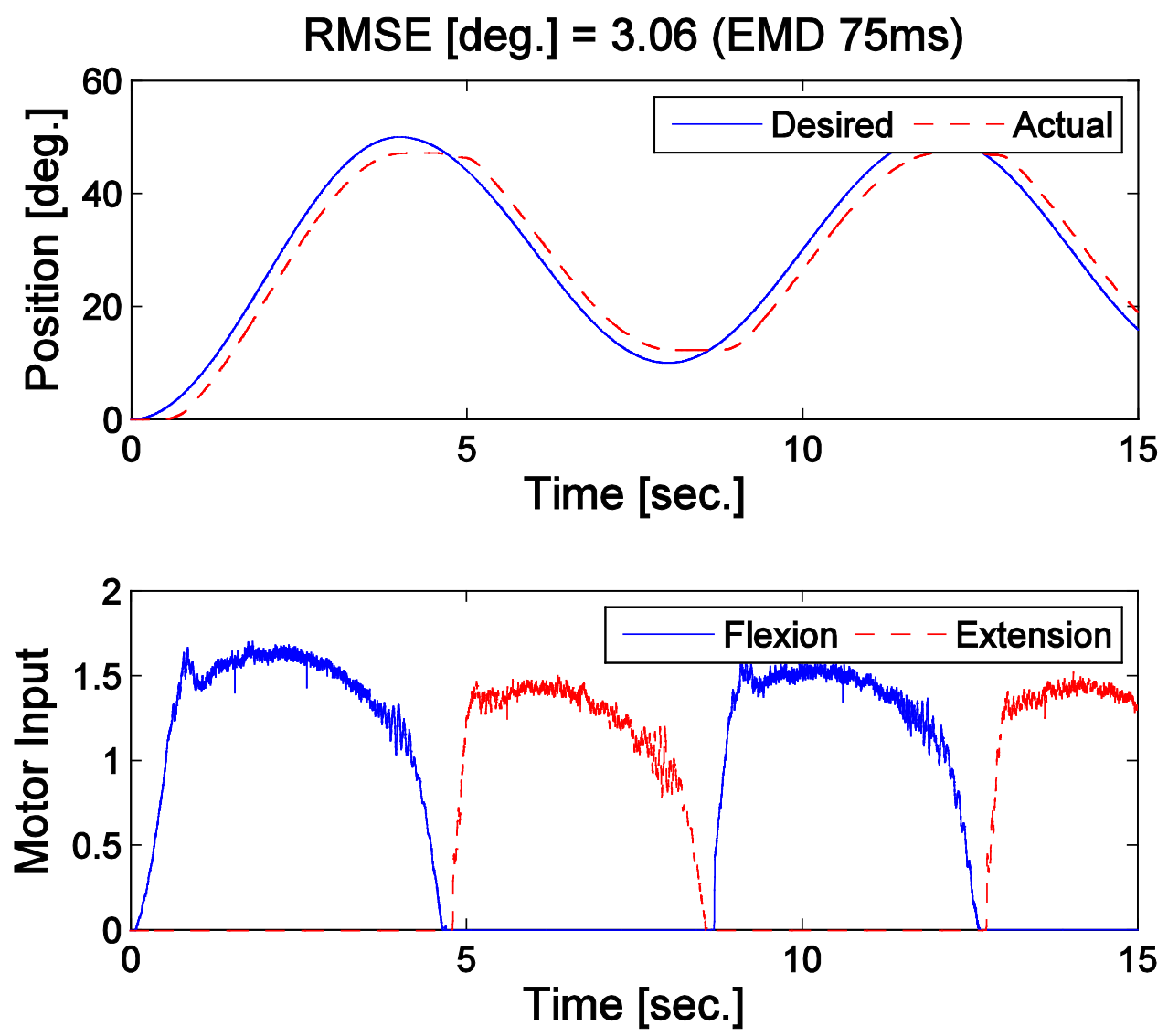

Figure 17. Trajectory and Motor Input (u1,u2) Plot from Dual Motors Experiment (75ms) 

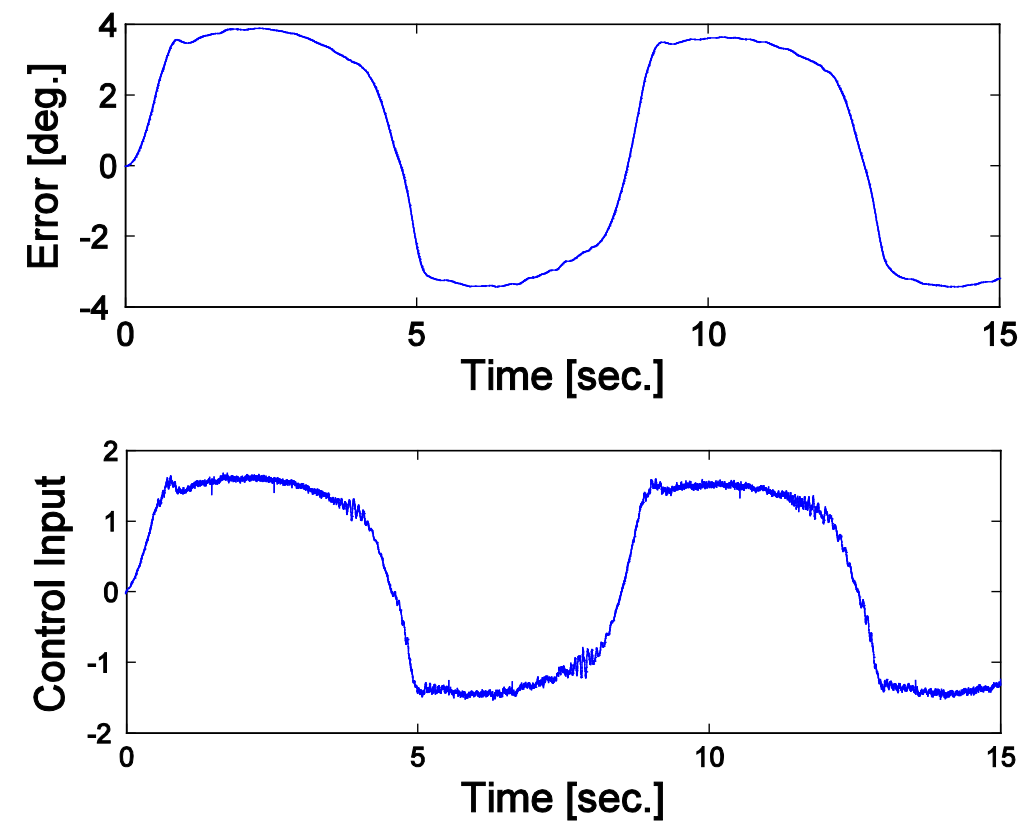

Figure 18. Error and Control Input ( v ) Plot from Dual Motors Experiment (75ms)
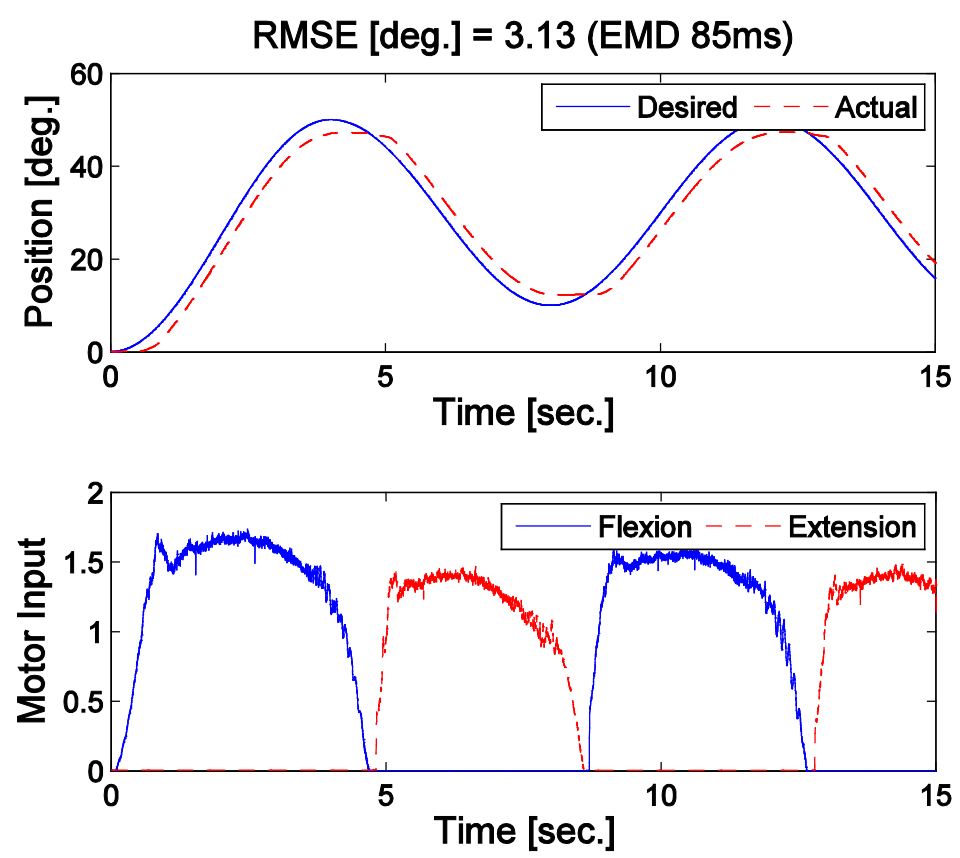

Figure 19. Trajectory and Motor Input (u1,u2) Plot from Dual Motors Experiment (85ms) 

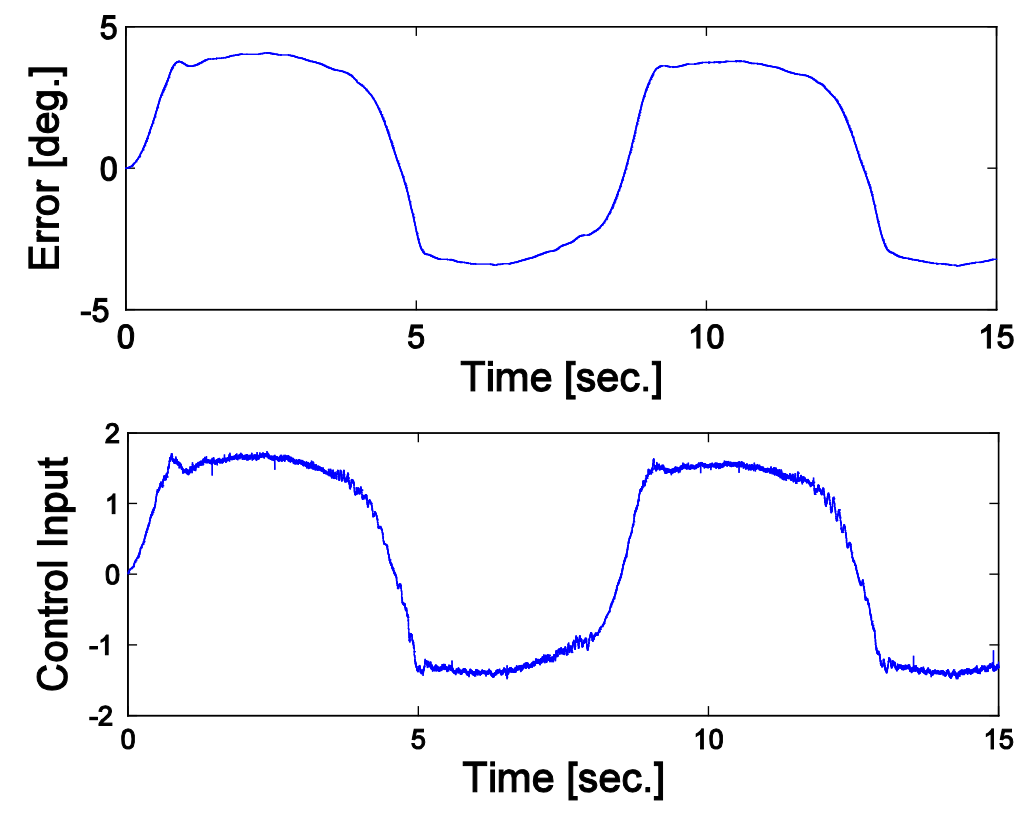

Figure 20. Error and Control Input ( v ) Plot from Dual Motors Experiment (85ms)

Table 3. RMSE of Experiments and Simulations with Different EMD Value

\begin{tabular}{|c|c|c|c|}
\hline Estimated EMD Value (ms) & $\begin{array}{c}\text { RMSE (deg.) } \\
\text { Dual }\end{array}$ & $\begin{array}{c}\text { RMSE (deg.) } \\
\text { Single }\end{array}$ & $\begin{array}{c}\text { RMSE (deg.) } \\
\text { Simulation }\end{array}$ \\
\hline 80 (Matched) & 2.84 & 2.79 & 2.58 \\
\hline 75 & 3.05 & 3.03 & 2.97 \\
\hline 85 & 3.13 & 3.06 & 2.70 \\
\hline
\end{tabular}

Table and plots above demonstrated the performance of the controller when applying to the dual motors testbed. The RMSE values increased compared to the simulations and single motor tests. Oscillations also occur as previous single motor tests when EMD in the compensator and the system are matched. Similar to the previous simulations and experiments, when the EMD value are not precisely matched with the time delay of the system, RMSE rose slightly, but still 
in a relatively small range, and the system remains stable. From the results, it can be concluded that this controller has satisfactory tracking performance when applying to the dual motors tests. 


\subsection{CONCLUSION AND FUTURE WORK}

\subsection{CONCLUSION}

In this thesis, a novel NMES switching controller with a predictive term that could compensate for EMDs is designed for antagonistic muscles. Lyapunov-based stability analysis proved semiglobally uniformly ultimately bounded tracking for the musculoskeletal system. The controller provided an arbitrary short transition period when two antagonistic muscles are simultaneously activated to ensure that there will be no discontinuities in muscle response so that the limb could flex and extend smoothly. The simulation result indicates that the controller is robust and could switch between opposing muscles without affecting the tracking performance. Further experiments using single servo motor and dual servo motors proved its performance when unmodeled nonlinearity and disturbance were introduced into the system.

\subsection{FUTURE WORK}

Future studies will focus on experimentally validating the newly developed controller on the human subjects. Figures below are demonstrations of the future human NMES experiment. 


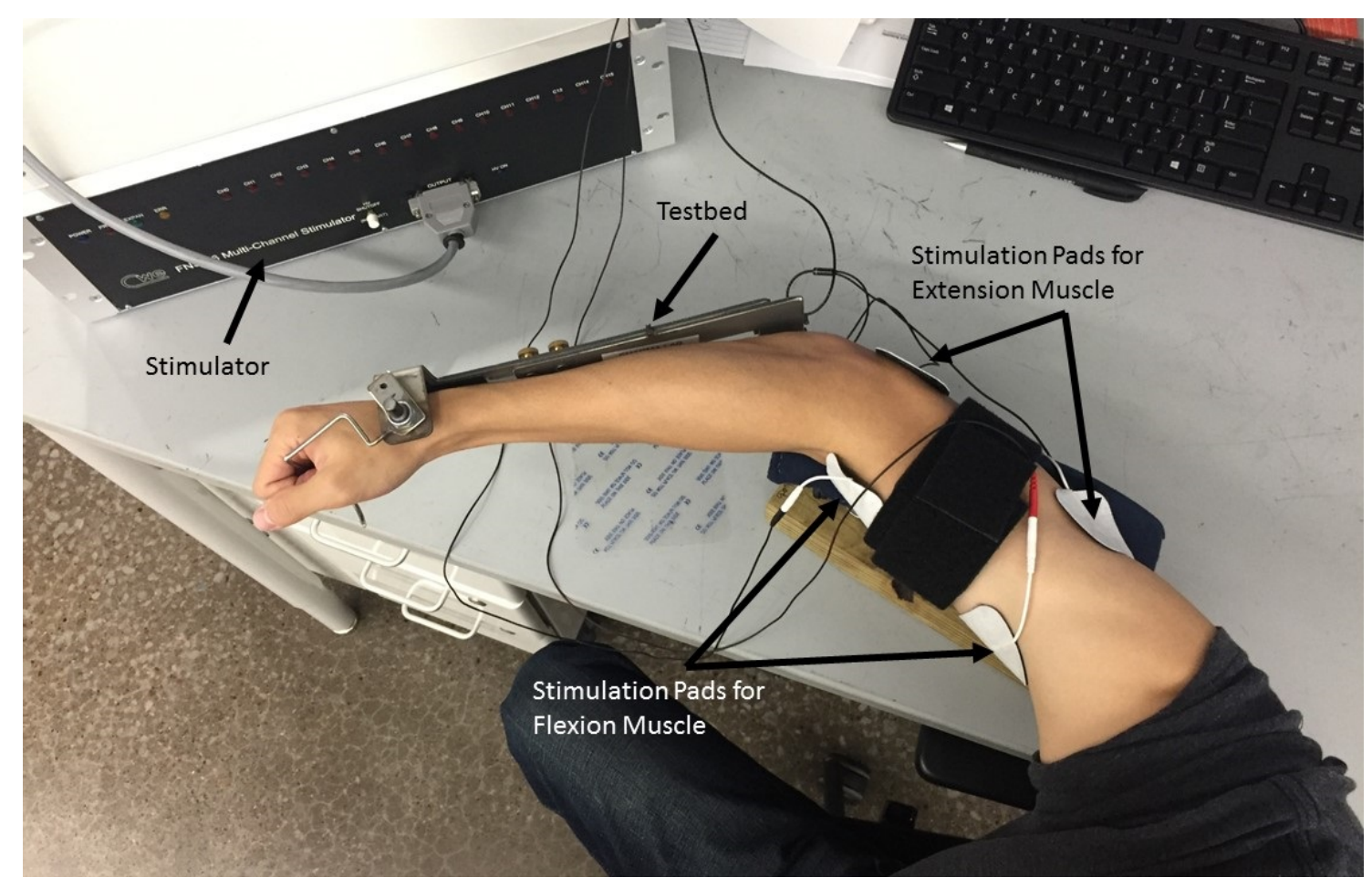

Figure 21. Demonstration of Planned Human NMES Experiments 1

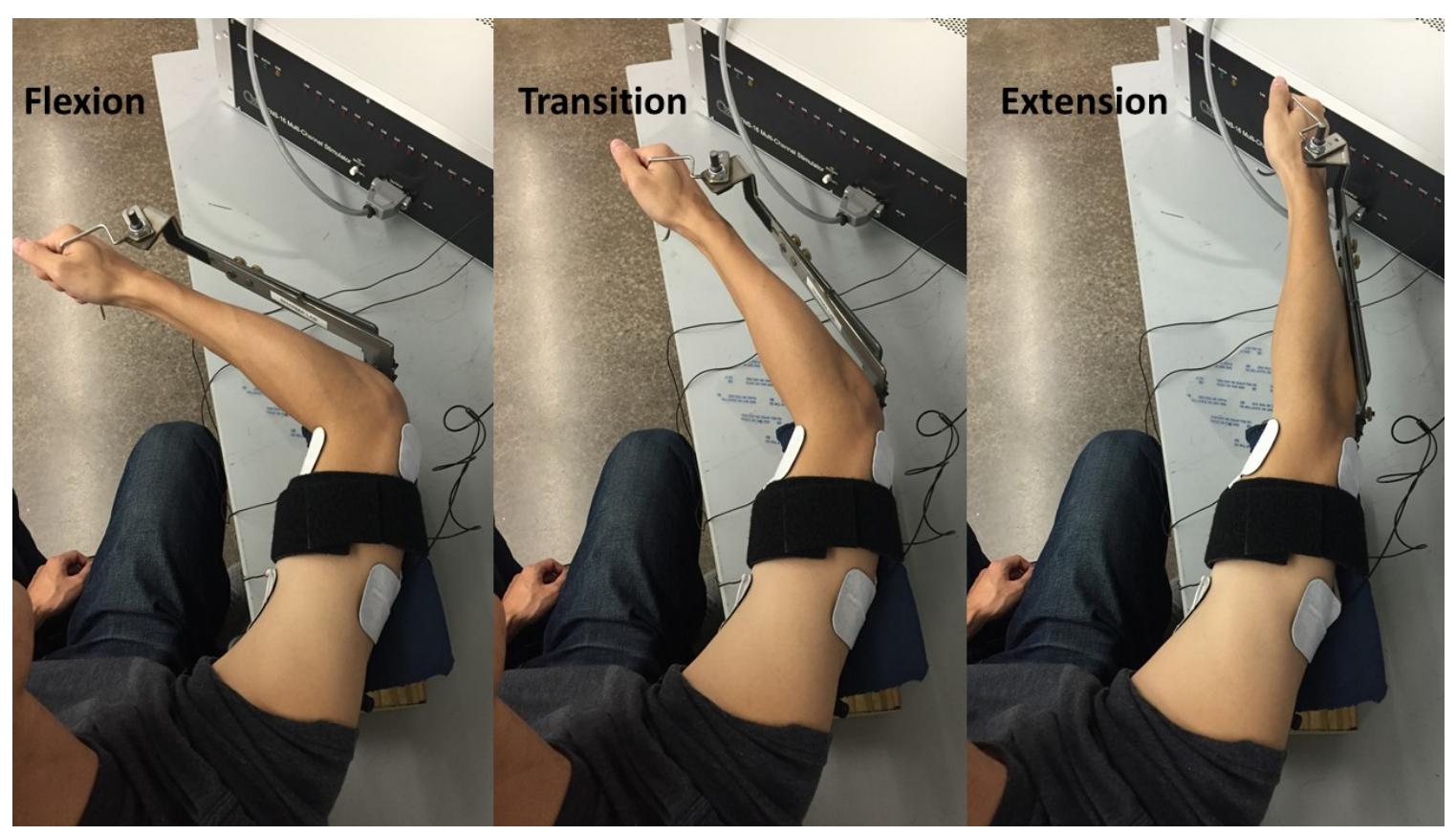

Figure 22. Demonstration of Planned Human NMES Experiments 2 
Besides experiments on human subjects, future work also includes making improvements to the controller. In this thesis, the controller is a PD-DC controller. Although it gave satisfactory tracking performance, improvements could be made to achieve lower RMS error. In [18], the PID-DC controller was demonstrated to give better tracking ability in unidirectional limb movements. The controller in this thesis can be improved by introducing an integral component just like in PID-DC controller into the system.

Another direction for future work is the compensation for unknown varying EMDs, as the EMD will increase as muscle fatigues and the NMES tends to induce significant fatigue rather than volitional contractions [49]. Results such as [49-51], have presented some methods for compensating varying unknown delay. The performance of the antagonist muscle controller will be further improved if techniques for compensating varying delays could be adapted to it. 


\section{APPENDIX A}

\section{SIMULINK BLOCK DIAGRAMS FOR SIMULATIONS \& EXPERIMENTS}

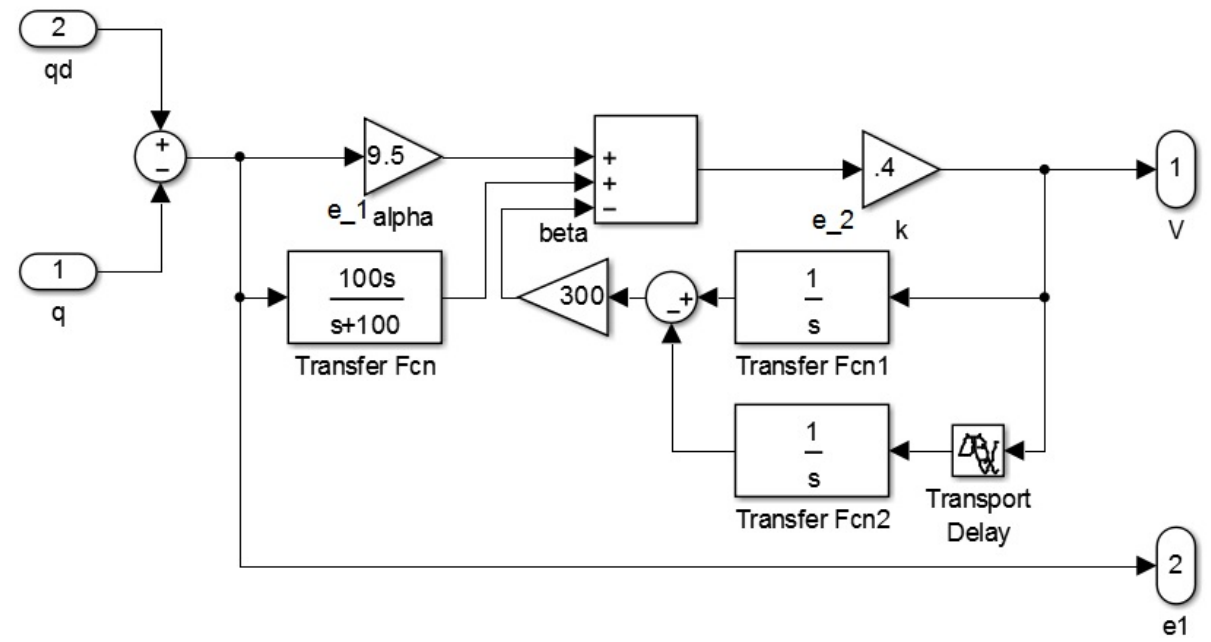

Figure 23. Simulink Block Diagram of Controller 


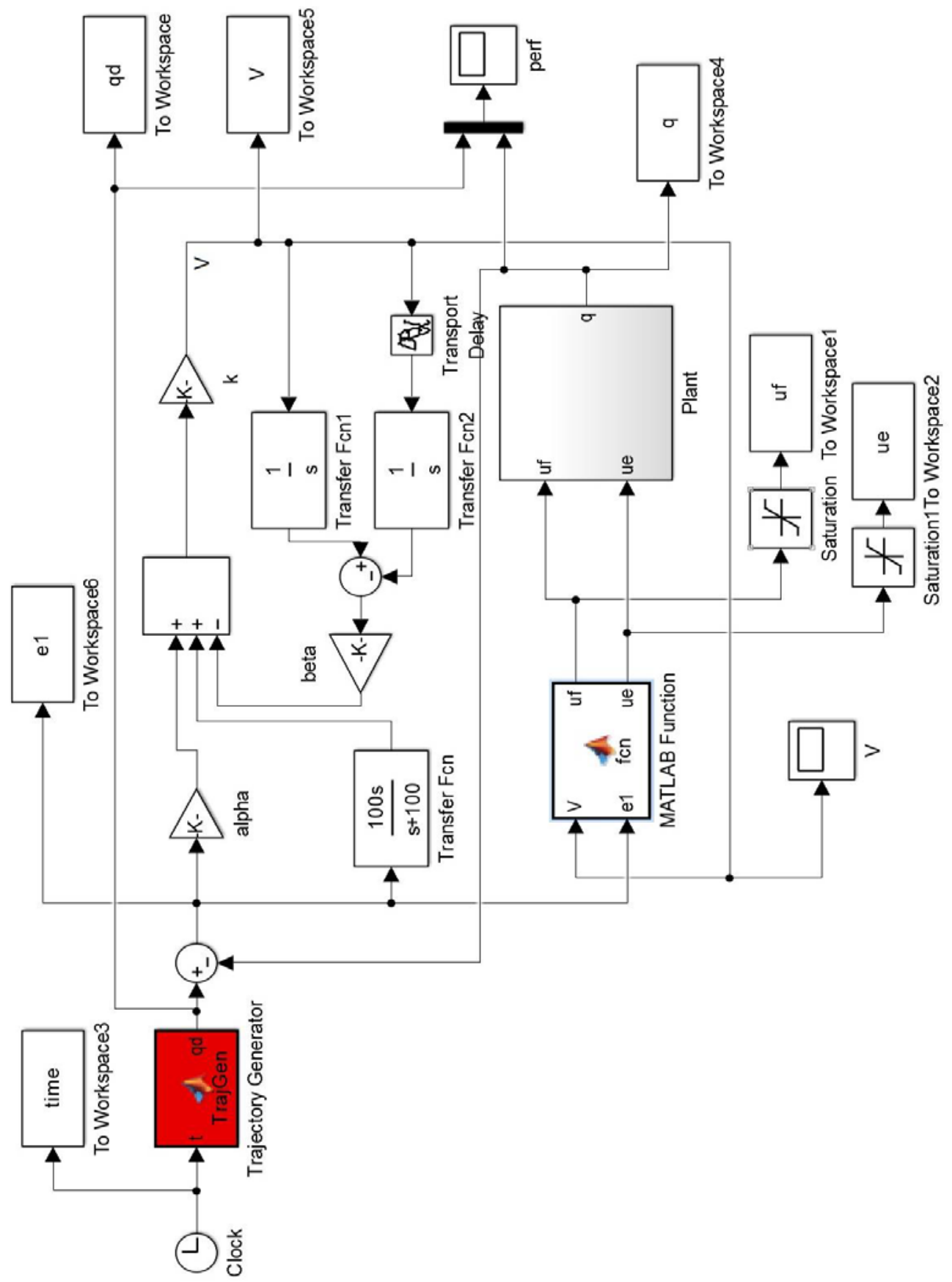

Figure 24. Simulink Block Diagram for Simulation 


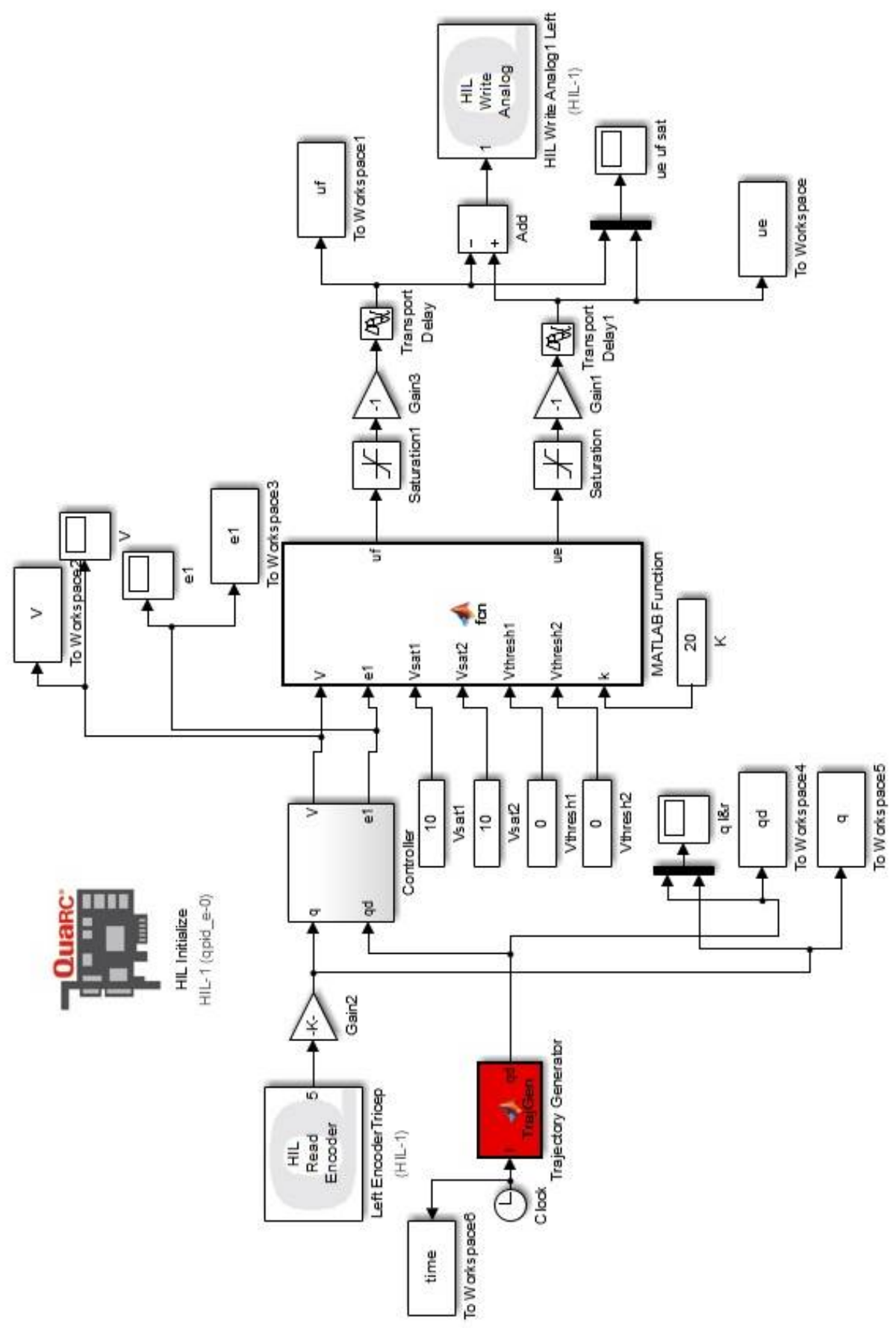

Figure 25. Simulink Block Diagram for Single Motor Experiment 


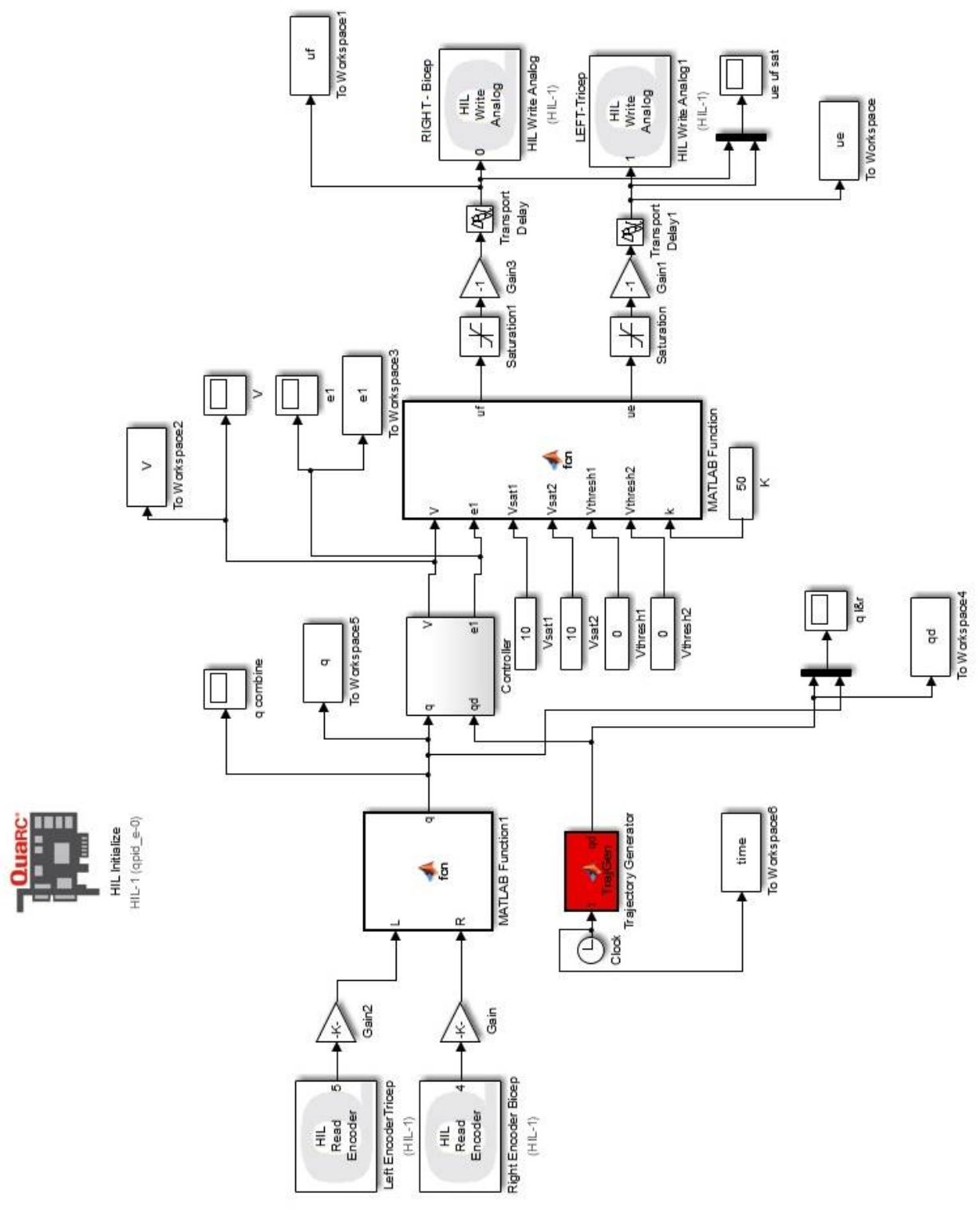

Figure 26. Simulink Block Diagram for Dual Motors Experiment 


\section{BIBLIOGRAPHY}

[1] Peckham, P.H. and J.S. Knutson, Functional Electrical Stimulation for Neuromuscular Applications*. Annu. Rev. Biomed. Eng., 2005. 7: p. 327-360.

[2] Nekoukar, V. and A. Erfanian, A decentralized modular control framework for robust control of FES-activated walker-assisted paraplegic walking using terminal sliding mode and fuzzy logic control. Biomedical Engineering, IEEE Transactions on, 2012. 59(10): p. 2818-2827.

[3] Sharma, N., V. Mushahwar, and R. Stein, Dynamic optimization of FES and orthosisbased walking using simple models. Neural Systems and Rehabilitation Engineering, IEEE Transactions on, 2014. 22(1): p. 114-126.

[4] Jezernik, S., R.G. Wassink, and T. Keller, Sliding mode closed-loop control of FES controlling the shank movement. Biomedical Engineering, IEEE Transactions on, 2004. 51(2): p. 263-272.

[5] Sharma, N., C.M. Gregory, M. Johnson, and W.E. Dixon, Closed-loop neural networkbased NMES control for human limb tracking. Control Systems Technology, IEEE Transactions on, 2012. 20(3): p. 712-725.

[6] Schauer, T., N.-O. Negård, F. Previdi, K. Hunt, M. Fraser, E. Ferchland, and J. Raisch, Online identification and nonlinear control of the electrically stimulated quadriceps muscle. Control Engineering Practice, 2005. 13(9): p. 1207-1219.

[7] Sharma, N., K. Stegath, C.M. Gregory, and W.E. Dixon, Nonlinear neuromuscular electrical stimulation tracking control of a human limb. IEEE Transactions on Neural Systems and Rehabilitation Engineering, 2009. 17(6): p. 576-584.

[8] Sharma, N., P. Patre, C. Gregory, and W. Dixon. Nonlinear control of NMES: Incorporating fatigue and calcium dynamics. in ASME 2009 Dynamic Systems and Control Conference. 2009. American Society of Mechanical Engineers. 
[9] Downey, R.J., T.-H. Cheng, and W.E. Dixon. Tracking control of a human limb during asynchronous neuromuscular electrical stimulation. in Decision and Control (CDC), 2013 IEEE 52nd Annual Conference on. 2013. IEEE.

[10] Thrasher, T.A., V. Zivanovic, W. McIlroy, and M.R. Popovic, Rehabilitation of reaching and grasping function in severe hemiplegic patients using functional electrical stimulation therapy. Neurorehabilitation and neural repair, 2008. 22(6): p. 706-714.

[11] Chadwick, E., D. Blana, J. Simeral, J. Lambrecht, S.-P. Kim, A. Cornwell, D. Taylor, L. Hochberg, J. Donoghue, and R. Kirsch, Continuous neuronal ensemble control of simulated arm reaching by a human with tetraplegiaThis paper was originally submitted for the special issue containing contributions from the Fourth International BrainComputer Interface Meeting. Journal of neural engineering, 2011. 8(3): p. 034003.

[12] Schearer, E.M., Y.-W. Liao, E.J. Perreault, M.C. Tresch, W.D. Memberg, R.F. Kirsch, and K.M. Lynch, Multi-muscle FES force control of the human arm for arbitrary goals. Neural Systems and Rehabilitation Engineering, IEEE Transactions on, 2014. 22(3): p. 654-663.

[13] Previdi, F., M. Ferrarin, S.M. Savaresi, and S. Bittanti, Gain scheduling control of functional electrical stimulation for assisted standing up and sitting down in paraplegia: a simulation study. International Journal of Adaptive Control and Signal Processing, 2005. 19(5): p. 327-338.

[14] Gollee, H., K.J. Hunt, and D.E. Wood, New results in feedback control of unsupported standing in paraplegia. Neural Systems and Rehabilitation Engineering, IEEE Transactions on, 2004. 12(1): p. 73-80.

[15] Hunt, K.J., M. Munih, and N.d.N. Donaldson, Feedback control of unsupported standing in paraplegia. I. Optimal control approach. Rehabilitation Engineering, IEEE Transactions on, 1997. 5(4): p. 331-340.

[16] Nekoukar, V. and A. Erfanian. Adaptive terminal sliding mode control of ankle movement using functional electrical stimulation of agonist-antagonist muscles. in Engineering in Medicine and Biology Society (EMBC), 2010 Annual International Conference of the IEEE. 2010. IEEE.

[17] Benoussaad, M., K. Mombaur, and C. Azevedo-Coste. Nonlinear model predictive control of joint ankle by electrical stimulation for drop foot correction. in Intelligent Robots and Systems (IROS), 2013 IEEE/RSJ International Conference on. 2013. IEEE. 
[18] Alibeji, N., N. Kirsch, S. Farrokhi, and N. Sharma, Further results on predictor-based control of neuromuscular electrical stimulation. Neural Systems and Rehabilitation Engineering, IEEE Transactions on, 2015. 23(6): p. 1095-1105.

[19] Sharma, N., C.M. Gregory, and W.E. Dixon, Predictor-based compensation for electromechanical delay during neuromuscular electrical stimulation. Neural Systems and Rehabilitation Engineering, IEEE Transactions on, 2011. 19(6): p. 601-611.

[20] Masani, K., A.H. Vette, N. Kawashima, and M.R. Popovic, Neuromusculoskeletal torque-generation process has a large destabilizing effect on the control mechanism of quiet standing. Journal of neurophysiology, 2008. 100(3): p. 1465-1475.

[21] Sharma, N., S. Bhasin, Q. Wang, and W.E. Dixon, Predictor-based control for an uncertain Euler-Lagrange system with input delay. Automatica, 2011. 47(11): p. 23322342.

[22] Freeman, C., D. Tong, K. Meadmore, A. Hughes, E. Rogers, and J. Burridge. FES based rehabilitation of the upper limb using input/output linearization and ILC. in American Control Conference (ACC), 2012. 2012. IEEE.

[23] Freeman, C.T., A.-M. Hughes, J.H. Burridge, P.H. Chappell, P.L. Lewin, and E. Rogers, A model of the upper extremity using FES for stroke rehabilitation. Journal of biomechanical engineering, 2009. 131(3): p. 031011.

[24] Meadmore, K.L., T.A. Exell, E. Hallewell, A.-M. Hughes, C.T. Freeman, M. Kutlu, V. Benson, E. Rogers, and J.H. Burridge, The application of precisely controlled functional electrical stimulation to the shoulder, elbow and wrist for upper limb stroke rehabilitation: a feasibility study. Journal of neuroengineering and rehabilitation, 2014. 11(1): p. 1.

[25] Sampson, P., C. Freeman, S. Coote, S. Demain, P. Feys, K. Meadmore, and A.-M. Hughes, Using functional electrical stimulation mediated by iterative learning control and robotics to improve arm movement for people with multiple sclerosis. 2015.

[26] Alon, G., A.F. Levitt, and P.A. McCarthy, Functional electrical stimulation enhancement of upper extremity functional recovery during stroke rehabilitation: a pilot study. Neurorehabilitation and neural repair, 2007. 21(3): p. 207-215. 
[27] Chan, M.K.-l., R.K.-y. Tong, and K.Y.-k. Chung, Bilateral upper limb training with functional electric stimulation in patients with chronic stroke. Neurorehabilitation and Neural Repair, 2009. 23(4): p. 357-365.

[28] Schmidt, R.A. and T. Lee, Motor control and learning. 1988: Human kinetics.

[29] Wikipedia. Action Potential. 2016; Available from: https://en.wikipedia.org/wiki/Action_potential.

[30] Popovic, D. and T. Sinkjaer, Control of movement for the physically disabled: control for rehabilitation technology. 2012: Springer Science \& Business Media.

[31] Peckham, P.H., Functional electrical stimulation: current status and future prospects of applications to the neuromuscular system in spinal cord injury. Spinal Cord, 1987. 25(3): p. 279-288.

[32] Association, C.P. Canadian Paraplegic Association. 2006; Available from: http://canparaplegic.org/en/.

[33] Lynch, C.L. and M.R. Popovic, Functional electrical stimulation. Control Systems, IEEE, 2008. 28(2): p. 40-50.

[34] G. BROEKS, J., G. Lankhorst, K. Rumping, and A. Prevo, The long-term outcome of arm function after stroke: results of a follow-up study. Disability and rehabilitation, 1999. 21(8): p. 357-364.

[35] Bobath, B., Adult hemiplegia: evaluation and treatment. 1990: Elsevier Health Sciences.

[36] Langhammer, B. and J.K. Stanghelle, Bobath or motor relearning programme? A comparison of two different approaches of physiotherapy in stroke rehabilitation: a randomized controlled study. Clinical rehabilitation, 2000. 14(4): p. 361-369.

[37] Woldag, H. and H. Hummelsheim, Evidence-based physiotherapeutic concepts for improving arm and hand function in stroke patients. Journal of neurology, 2002. 249(5): p. 518-528. 
[38] Fasoli, S.E., H.I. Krebs, J. Stein, W.R. Frontera, and N. Hogan, Effects of robotic therapy on motor impairment and recovery in chronic stroke. Archives of physical medicine and rehabilitation, 2003. 84(4): p. 477-482.

[39] Robbins, S.M., P.E. Houghton, M.G. Woodbury, and J.L. Brown, The therapeutic effect of functional and transcutaneous electric stimulation on improving gait speed in stroke patients: a meta-analysis. Archives of physical medicine and rehabilitation, 2006. 87(6): p. 853-859.

[40] de Kroon, J.R., M.J. IJzerman, J. Chae, G.J. Lankhorst, and G. Zilvold, Relation between stimulation characteristics and clinical outcome in studies using electrical stimulation to improve motor control of the upper extremity in stroke. 2005.

[41] Glanz, M., S. Klawansky, W. Stason, C. Berkey, and T.C. Chalmers, Functional electrostimulation in poststroke rehabilitation: a meta-analysis of the randomized controlled trials. Archives of physical medicine and rehabilitation, 1996. 77(6): p. 549553.

[42] McCabe, J., M. Monkiewicz, J. Holcomb, S. Pundik, and J.J. Daly, Comparison of Robotics, Functional Electrical Stimulation, and Motor Learning Methods for Treatment of Persistent Upper Extremity Dysfunction After Stroke: A Randomized Controlled Trial. Archives of physical medicine and rehabilitation, 2015. 96(6): p. 981-990.

[43] Zhou, S., D.L. Lawson, W.E. Morrison, and I. Fairweather, Electromechanical delay in isometric muscle contractions evoked by voluntary, reflex and electrical stimulation. European journal of applied physiology and occupational physiology, 1995. 70(2): p. 138-145.

[44] Cavanagh, P.R. and P.V. Komi, Electromechanical delay in human skeletal muscle under concentric and eccentric contractions. European journal of applied physiology and occupational physiology, 1979. 42(3): p. 159-163.

[45] Smith, O.J., A controller to overcome dead time. iSA journal, 1959. 6(2): p. 28-33.

[46] Popović, L.Z. and N.M. Malešević. Muscle fatigue of quadriceps in paraplegics: comparison between single vs. multi-pad electrode surface stimulation. in Engineering in Medicine and Biology Society, 2009. EMBC 2009. Annual International Conference of the IEEE. 2009. IEEE. 
[47] Artstein, Z., Linear systems with delayed controls: a reduction. Automatic Control, IEEE Transactions on, 1982. 27(4): p. 869-879.

[48] Downey, R.J., T.-H. Cheng, M.J. Bellman, and W.E. Dixon. Switched tracking control of a human limb during asynchronous neuromuscular electrical stimulation. in American Control Conference (ACC), 2015. 2015. IEEE.

[49] Downey, R., R. Kamalapurkar, N. Fischer, and W. Dixon, Compensating for fatigueinduced time-varying delayed muscle response in neuromuscular electrical stimulation control, in Recent Results on Nonlinear Delay Control Systems. 2016, Springer. p. 143161.

[50] Yue, D. and Q.-L. Han, Delayed feedback control of uncertain systems with time-varying input delay. Automatica, 2005. 41(2): p. 233-240.

[51] Bekiaris-Liberis, N. and M. Krstic. Compensation of time-varying input delay for nonlinear systems. in Control \& Automation (MED), 2011 19th Mediterranean Conference on. 2011. IEEE. 